\title{
Dynamic Marketing Mix Allocation for Long-Term
}

\author{
Profitability
}

Ricardo Montoya*

Oded Netzer

Kamel Jedidi

December 16, 2007

* Ricardo Montoya is a doctoral candidate at the Graduate School of Business, Columbia University (email: rm2183@columbia.edu). Oded Netzer is an Assistant Professor of Marketing at the Graduate School of Business, Columbia University (email: on2110@columbia.edu). Kamel Jedidi is a Professor of Marketing at the Graduate School of Business, Columbia University (email: kj7@columbia.edu). Please address all correspondence to Ricardo Montoya, Graduate School of Business, Columbia University, 3022 Broadway, 311 Uris Hall, New York, NY 10027, USA. 


\begin{abstract}
To optimally allocate its marketing mix across customers, a firm needs to consider the evolution of its customers over time. Changes in the marketing environment, as well as intrinsic changes in preferences or needs, may discretely shift customers into different buying-behavior states. The ability to identify the dynamics in customer behavior and its drivers presents an opportunity for the firm to influence the movement of customers to more favorable states of buying behavior. Accordingly, we address the following managerial questions in this research: (1) how can the firm dynamically segment its customer base? (2) what are the short- and long-term effects of marketing activities? and (3) how should the firm allocate and target its marketing resources to maximize long-term profitability?

To address these questions we propose a non-homogeneous hidden Markov model that accounts for dynamics in customer behavior, the long-term impact of marketing actions, and customer heterogeneity. We capture dynamics in customer behavior by allowing customers to transition over time among a set of latent states of buying behavior. We develop a unique and flexible approach to capture the enduring effect of marketing actions by incorporating a non-stationary transition matrix that is dynamically affected by these actions. To optimally allocate marketing activities, we formulate a dynamic programming approach which takes into account the evolution of customers' behavior.

We apply the model in the context of direct-to-physicians marketing in a major pharmaceutical company. The results suggest that physicians transition among three behavioral states over time, showing a high degree of dynamics. Furthermore, the direct-to-physician marketing activities have varying degrees of short- and long-term effects that depend on the physician's prescription-behavior state. Specifically, we find that (i) both detailing and sampling have mostly long-term effects; (ii) detailing and sampling have a total duration impact of approximately 10 and 5 months, respectively; (iii) detailing is most effective as an acquisition marketing tool, whereas sampling is most effective as a retention tool.

Using a counterfactual analysis, the optimization results show that by applying our dynamic marketing allocation approach, the firm could increase the number of prescriptions and its profits by as much as $51 \%$ and $80 \%$, respectively. Moreover, our analysis suggests that the pharmaceutical firm should decrease its current detailing and sampling efforts by $30 \%$ and $20 \%$, respectively. The integrative framework we propose provides important marketing implications for managing customers and maximizing long-run profitability.
\end{abstract}

Keywords: marketing mix allocation, long-term effect of marketing activities, hidden Markov model, Bayesian estimation, dynamic programming, pharmaceutical marketing. 


\section{Introduction}

"The pharmaceutical industry is under significant pressure to consider its costs very carefully. Since marketing budgets often represent a major proportion of a company's cost base, they can easily become the target of budget cuts. Although marketing investments are profitable, in the main, returns are now under intense scrutiny with all budgets being squeezed. The pressure to measure marketing return and effectiveness has never been stronger. Currently, much budget is spent despite marketers being unable to identify which combination of activities has the greatest growth potential, and without knowing what specific effect individual activities are having on physicians over time."

- Andrée Bates (2006)

In order to stay competitive, firms need to wisely allocate marketing mix resources with the objective of establishing and sustaining long-term relationships with their customers. In doing so, managers should consider how a given marketing budget should be allocated across different marketing activities, customers, and time. However, marketing resource allocation decisions are complicated and managers tend to address them with fairly arbitrary simplified heuristics and decision rules (Mantrala 2002). Such rules usually result in sub-optimal decisions, which could lead to a significant waste of resources. Improving these decisions could have a direct impact on long-term profitability.

When making marketing allocation decisions, firms often face the problem of trading off between short- and long-term revenues. For example, Harrah's, through its Total Rewards loyalty program, may offer a free dinner to customers who are having a "bad day". Such a marketing action pulls gamblers away from the casino, producing a short-term loss of revenues. In doing so, however, the casino expects to bring future revenues by shifting the customers to a more favorable state of relationship with Harrah's. Similarly, Procter $\&$ Gamble faces such trade-offs when giving free samples of Pampers diapers. Though this action cannibalizes current purchases, the company hopes to have a long-term impact by increasing its customer base with customers who otherwise would have not tried the product. American Airlines may offer to current customers either last-minute deals or free upgrades in order to fill empty first-class seats. A last-minute deal creates immediate revenues for the company, whereas a free upgrade produces an opportunity cost and additional service costs to current operations. In choosing the free upgrade, however, the company anticipates an increase in customer loyalty and long-term 
profits.

In these examples, a successful marketing mix allocation at the individual level could enhance customer equity and, therefore, constitutes an important tool for managing the firm's customer base. However, these examples demonstrate that the task of allocating resources at the individual level is complicated and requires the knowledge of individual customer behavior and responsiveness to marketing interventions over time. The resource allocation task becomes even more complicated when firms have multiple marketing interventions with varying degrees of short- and long-term effects, and when customers have evolving preferences and sensitivities.

Understanding customer dynamics and the varying impact of marketing activities is critical for optimizing marketing decisions. Ignoring such dynamics can result in misleading inferences regarding the temporal pattern of elasticities. A firm that is myopic and does not consider the long-term effect of its marketing interventions is likely to under allocate marketing interventions with relatively small short-term effects but substantial long-term effects, such as the ones described above. Similarly, a firm that is forward-looking in its marketing mix allocation, but does not capture well the evolution of customer preferences and buying behavior over time, is likely to allocate its marketing mix sub-optimally.

This paper proposes an integrative resource allocation approach that simultaneously considers a forward-looking, individual-level allocation of marketing resources, where customers' preferences change over time, and the firm can influence the dynamics in customers' preferences using multiple marketing interventions with both short- and long-term effects. Our approach is built around a non-homogeneous hidden Markov model (HMM) that accounts for dynamics in customer behavior and the long-term impact of marketing actions. We capture dynamics in customer behavior by allowing customers to transition over time among a set of latent states of buying behavior. We model the enduring impact of marketing actions by incorporating a non-homogeneous transition matrix that is dynamically affected by these actions. To optimally allocate and target marketing resources and to maximize long-term profitability, we propose a stochastic dynamic programming approach which takes into account customer heterogeneity, the evolution in customers' behavior, the short- and long-term effects of marketing interventions, and the long-term payoff.

This paper is organized as follows: Section 2 motivates the need to consider the dynamics in customer behavior and the short- and long-term effects of marketing actions in marketing 
mix resource allocation. Section 3 presents the modeling approach proposed for capturing the dynamics in customer-buying behavior, the response to marketing mix interventions, and the dynamic optimal marketing mix allocation. Section 4 illustrates the proposed model using direct-to-physicians marketing data from a major pharmaceutical company. Section 5 concludes by discussing practical implications, theoretical contributions, and future directions.

\section{Marketing Mix Allocation and Dynamic Customer Management}

In recent years there has been an increasing interest in the study of marketing mix allocation problems (Rust and Verhoef 2005). The customer relationship management (CRM) literature suggests that marketing initiatives should be evaluated by measuring their impact on the longterm value generated by improving the relationship with the customer. To do so, the firm must be able to measure the dynamics in customer buying behavior and the long-term impact of its marketing actions. Indeed, Rust and Chung (2006) highlight the need for an integrative framework that maximizes long-term profits, accounting for individually targeted marketing interventions and the joint effect of multiple marketing interventions. Our paper presents such a framework and contributes to the understanding of how and why customer profitability could change over time due to intrinsic buying-behavior dynamics and marketing interventions.

Accordingly, in this section we briefly review previous work related to the different components of our model: dynamics in customer buying behavior, long-term effect of marketing activities, and marketing mix allocation.

\subsection{Dynamics in Customer Buying Behavior}

Several approaches have been suggested to model customer evolution over time. Most of these use observed variables to capture the dynamics in buying behavior. In the CRM literature, heterogeneity and dynamics in customer behavior are often captured using the recency, frequency, and monetary value (RFM) framework (e.g., Bitran and Mondschein 1996; Colombo and Jiang 1999; Pfeifer and Carraway 2000). RFM analysis is a deterministic approach in which customers are characterized by how recently they transacted, how frequently they have transacted in the past, and the value of those transactions. This approach is commonly used in the industry due 
to its ability to capture dynamics in customer behavior in a relatively simple way. For example, Pfeifer and Carraway (2000) model customer relationship evolution as a Markov chain, in which customers transition among several observed states characterized by the RFM variables. While the RFM approach is useful for a dynamic descriptive segmentation of the firm's customer base, it is not obvious how it can be used to capture the dynamic and enduring effects of marketing actions and to optimize marketing allocation, which are key objectives in our study.

Another common approach to modeling customer evolution using observed states is the state-dependence model (e.g., Bucklin and Lattin 1991; Guadagni and Little 1983; Heckman 1981; Srinivasan and Kesavan 1976). Our proposed HMM extends the state-dependence model by allowing the states to be defined not only by past behavior, but also by external factors such as marketing activities. Ignoring such external effects can lead to overestimation of statedependence (Erdem and Sun 2001; Keane 1997). Most models of state-dependence ignore the long-term effect of marketing variables and are therefore susceptible to this bias. Conversely, a model that estimates the long-term effect of marketing activities but ignores state-dependence may overestimate the total effect of marketing interventions. To overcome these limitations and to simultaneously capture the internal and external sources of dynamics in buying behavior, we propose a hidden Markov model (HMM; see McDonald and Zucchini 1997 and Rabiner 1989 for a detailed review of HMMs).

There is a small, but increasing, number of applications of HMMs in marketing. For example, Liechty et al. (2003) develop a HMM to identify the respondents' attentional states when they are exposed to an advertisement viewing task. Montgomery et al. (2004) build a continuous-time, two-state HMM to investigate how customers move among different categories of web pages when searching for information online. Du and Kamakura (2006) present a HMM to capture the dynamic evolution of American families' lifecycles. Moon et al. (2007) use a HMM to model unobserved competitors' marketing actions in a pharmaceutical context. Netzer et al. (2007) analyze the dynamic relationship between alumni and their university, allowing for different states of relationship with the university which affect donation behavior. With the exception of Netzer et al. (2007), the applications above assume a homogeneous HMM characterized by a stationary transition matrix. In our framework, allowing for a non-stationary transition matrix is crucial since it allows one to capture the enduring effects of marketing activities, and disentangle them from the short-term effects. Unlike Netzer et al. (2007) - who do 
not incorporate firm's initiated marketing actions - our model captures the effect of marketing interventions on customer dynamics and derives an optimal marketing allocation strategy for these interventions.

\subsection{Short- and Long-Term Effects of Marketing Activities}

Customers may exhibit dynamics not only due to the intrinsic evolution in their buyingbehavior, but also due to the long-term impact of the firm's actions. Furthermore, to optimally allocate marketing activities, firms need to consider both customer dynamics and the shortand long-term effects of their actions.

One can divide the literature analyzing the long-term effect of marketing actions into mass marketing and direct marketing. Several studies have analyzed the long-term effect of mass marketing activities such as advertising and price promotions (Dekimpe and Hanssens 1995, 1999; Dekimpe et al. 2005; Jedidi et al. 1999; Mela et al. 1997). Most of these studies assume a stable market (Mela et al. 1997; Papatla and Krishnamurthi 1996) and use a Koyck-type model, which implies that performance will return to its pre-marketing intervention level. Dekimpe and Hanssens (1995) and Dekimpe et al. (2005), on the other hand, have examined the effect of marketing actions in evolving markets using a persistence modeling approach to capture longterm changes in performance. Since the studies above investigate the short- and long-term effect of mass marketing actions, the issue of optimal allocation and targeting of these activities is rarely addressed.

In contrast, most of the direct marketing literature is interested in the targeting and allocation of marketing activities (Bitran and Mondschein 1996; Elsner et al. 2004; Gönül and Ter Hofstede 2006; Roberts and Berger 1999). However, this literature often ignores the possibly long-term effects of the marketing interventions when solving the marketing allocation problem. Some exceptions can be found in the context of catalog mailing decisions. For instance, Gönül and Shi (1998) present a structural dynamic programming approach where customers optimize a dynamic Markov game, by contemplating how their purchase decisions will affect the firm's future marketing decisions. Simester et al. (2006) present a nonparametric approach to capture customers' dynamics and the long-term effect of marketing actions. They provide a dynamic programming problem that the firm solves in order to mail catalogs to its customers. Our paper extends these papers by presenting a non-homogeneous HMM framework to capture the long-term effects of multiple marketing actions and their influence on customer dynamics. Our 
approach pushes forward the literature by allowing us to disentangle short-term and long-term effects, and take advantage of their influence at the individual level when allocating marketing resources.

\subsection{Marketing Mix Allocation}

The marketing mix allocation literature could generally be divided based on the portfolio of marketing mix interventions allocated and the level of aggregation in allocating the marketing mix. The direct marketing literature often deals with a single marketing activity such as catalog mailing (Bitran and Mondschein 1996; Gönül and Shi 1998; Gönül and Ter Hofstede 2006; Simester et al. 2006), optimal pricing decisions (Lewis 2005), and couponing decisions (Bawa and Shoemaker 1987; Rossi et al. 1996). These studies often deal with optimization without budget constraints when making such allocation decisions. Mass marketing mix allocation studies, on the other hand, typically consider a pair of marketing actions, with or without their synergies. For example, advertising and promotion (Jedidi et al. 1999; Mela et al. 1997, Naik et al. 2005), sales force and advertising (Gatignon and Hanssens 1987), or advertising between different media channels (Naik et al. 1998). In contrast to the direct marketing literature, these studies involve aggregate allocation of mass marketing mix interventions. Most of these studies do not account for changes in marketing mix sensitivity over time. In this paper, we extend the literature on marketing mix allocation by offering an integrative approach for managing customers through an optimal allocation of multiple, individually-targeted, marketing actions for long-run profitability. We do so, while taking into account heterogeneous changes in customer behavior and dynamic response to marketing interventions, and considering the possibility of budget constraints.

\section{Model Development}

To model the dynamics in buying behavior and the short- and long-term effects of marketing activities we build a HMM. In our model, customers can transition among a set of buyingbehavior states, where the transition between the states, as well as the buying behavior given a state, are affected by multiple marketing activities. We consider two aspects of customer heterogeneity: customers could differ in terms of their propensity of being in each of the states and in their response to marketing interventions. To optimally allocate marketing resources 
across customers, we solve a stochastic dynamic programming (DP) problem, accounting for customers' heterogeneity and dynamics, and maximizing customer value for a given planning horizon. We use a partially observed markov decision process (POMDP) to capture stochasticity in the customer's buying-behavior state membership. In this section, we first describe how our modeling approach captures customer dynamics through a HMM. We then explain how the HMM can capture both short- and long-term effects of marketing actions. We conclude this section with a description of how one can use our proposed model and the POMDP to optimize marketing mix allocation.

\subsection{Customer Dynamics: The Hidden Markov Model}

We assume a set of customers who make repeated purchases and are exposed to marketing activities initiated by the firm, both of which are observed by the researcher over time. We define a finite set of buying-behavior states that can be characterized by the intrinsic preference for the product, the purchase propensity, and the responsiveness to different marketing initiatives. For instance, let us assume two buying-behavior states. At the low buying-behavior state, customers make only few purchases, possibly due to the need to acquire information about the product. Consequently, customers in this state may be responsive to marketing initiatives that provide information (e.g., advertising and free samples). In contrast, customers at the higher buying-behavior state, which corresponds to high purchase frequency, are likely to be affected by marketing initiatives aimed at keeping customers engaged (e.g., loyalty programs and free upgrades). Furthermore, marketing initiatives not only affect customer behavior at each buying-behavior state, but may also trigger the transitions between these states. For example, free samples may induce customers to try the product and move them from the low buying-behavior state to the higher state. In contrast, increasing price or making it difficult for customers to redeem rewards in the loyalty program, may induce customers to switch from the high buying-behavior state to the lower one.

Buying-behavior states are often not observed and therefore need to be inferred from customers' observed behavior. Accordingly, we propose a HMM which is built of a set of latent buying-behavior states. Customers can stochastically transition among the buying-behavior states through a Markovian first-order process. The transitions between the states are a function of marketing mix activities. We relate the unobserved buying-behavior states to the observed choices through a state-dependent component, which captures the short-term effect of 
different marketing initiatives on buying behavior conditioned on the current customer state.

Throughout, we use four generic subscripts: $i$ denotes an individual customer $(i=1, \ldots, I)$, $j$ denotes a particular marketing activity $(j=1, \ldots, J), t$ denotes a time period $(t=1, \ldots, T)$, and $s$ denotes a buying-behavior state $(s=1, \ldots, S)$. We define customer $i$ 's decision at time $t$ by $Y_{i t}$. The customer decision could be about category incidence, brand choice, or quantity purchased, among others. We use $X_{i t} \in\{1, \ldots, S\}$ to denote customer $i$ 's state membership at time $t$, and $\mathbf{z}_{i t}=\left(z_{i t}^{1}, \ldots, z_{i t}^{J}\right)$ to represent a vector of covariates (e.g., marketing mix variables, demographics) for customer $i$ at period $t$.

Figure 1 illustrates the sequence of customer $i$ 's decisions and firm's actions in the proposed HMM. At period $t-1$ the customer is in the latent state $X_{i t-1}$. During period $t-1$ the firm decides to implement its marketing actions $\mathbf{z}_{i t-1}$, which have an immediate effect on the customer's decision at period $t-1$ and a delayed effect on the customer's transition to state $X_{i t}$ at period $t$. Specifically, given the current state $\left(X_{i t-1}\right)$ and marketing actions $\left(\mathbf{z}_{i t-1}\right)$, the customer decides $Y_{i t-1}$. Then, the customer moves from state $X_{i t-1}$ to state $X_{i t}$ with probability $q_{i t}$, which is a function of the marketing actions $\mathbf{z}_{i t-1}$. Finally, the customer is in state $X_{i t}$ at the beginning of period $t$, and the cycle continues. At this point, it is important to note that the researcher observes $Y_{i t}$ and $\mathbf{z}_{i t}$ for every period, but does not observe either the states $X_{i t}$ or the transition probabilities $q_{i t}$, which are the parameters of the HMM.

Figure 1: Sequence of customer's decisions and firm's actions

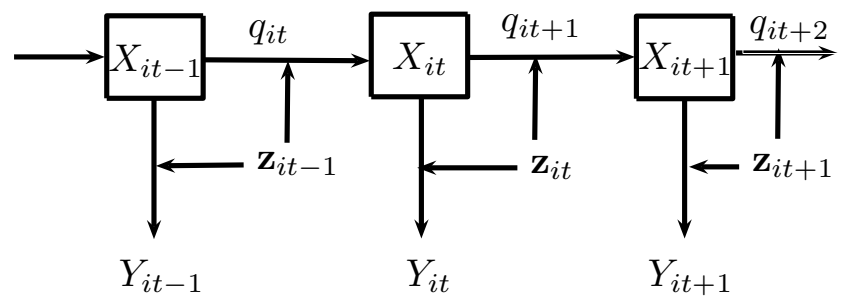

In the HMM, the joint probability of a sequence of decisions up to time $t\left\{Y_{i 1}=y_{i 1}, \ldots, Y_{i t}=\right.$ $\left.y_{i t}\right\}$, includes three main components: (1) the initial hidden states membership probabilities $\left(\boldsymbol{\pi}_{i}\right),(2)$ a sequence of probabilistic transitions among the buying-behavior states $\left(\mathbf{Q}_{i t}\right)$, and (3) a set of choice probabilities conditioned on the buying-behavior states $\left(\mathbf{M}_{i t}\right)$. Next, we describe our formulation of each one of the three model's components.

3.1.1. Initial State Membership Probabilities. The initial state membership probabilities can be treated as parameters to be estimated. We can write the Sx1 vector of initial buying- 
behavior state membership probabilities as $\pi_{i}^{\prime}=\left[\pi_{i 1}, \pi_{i 2}, \ldots, \pi_{i S}\right]$ where $0 \leq \pi_{i s} \leq 1$ and $\sum_{s=1}^{S} \pi_{i s}=1$. This leads to $S-1$ parameters to be estimated. Each of the initial probabilities $\pi_{i s}$ could be related to the covariates associated with the first period (e.g., demographic variables or acquisition marketing activities) as follows:

$$
\pi_{i s}=P\left(X_{i 1}=s \mid \mathbf{z}_{i 0}\right)
$$

where $X_{i 1}$ indicates customer $i$ 's state membership at time 1 , and $\mathbf{z}_{i 0}$ denotes the set of covariates known for customer $i$ at the first period.

3.1.2. The Markov Chain Transition Matrices. The transition matrix $\mathbf{Q}_{i t}$ governs the customer transitions among the states post period 1 . We model $\mathbf{Q}_{i t}$ at the individual level as a function of observed marketing initiatives and customer characteristics. Therefore, the non-homogeneous transition matrix for customer $i$ can be written as:

$$
\mathbf{Q}_{i t}=\left(\begin{array}{cccc}
q_{i 11 t} & q_{i 12 t} & \cdots & q_{i 1 S t} \\
\vdots & \ddots & & \vdots \\
q_{i S 1 t} & q_{i S 2 t} & \cdots & q_{i S S t}
\end{array}\right)
$$

Each element of the transition matrix, corresponding to the probability that customer $i$ switches from state $s^{\prime}$ to $s$ in period $t$, can be written as:

$$
q_{i s^{\prime} s t}=P\left(X_{i t}=s \mid X_{i t-1}=s^{\prime}, \mathbf{z}_{i t-1}\right),
$$

where $0 \leq q_{i s^{\prime} s t} \leq 1, \sum_{s=1}^{S} q_{i s^{\prime} s t}=1$. Thus, the propensity to transition from one state to another is a function of unobserved (to the researcher) factors, which can be captured by a transition random-effect coefficient, and a set of marketing actions in period $t-1$, which can affect the customer's transitions to a different behavioral-state in period $t$.

We follow Netzer et al. (2007) in parametrizing the non-homogeneous hidden state transitions as an ordered logit model (Greene 1997). Let $u_{i t}$ be a latent variable that captures customer $i$ 's transition propensity. We define $u_{i t}=\boldsymbol{\rho}_{i s}^{\prime} \cdot \mathbf{z}_{i t-1}+\epsilon_{i t}$, where $\boldsymbol{\rho}_{i s}$ is a vector of regression weights and $\epsilon_{i t}$ is an error term, i.i.d. extreme value. Higher values for $u_{i t}$ indicate higher likelihood to switch to (or to stay in) higher buying-behavior states.

Following the ordered logit model, we specify a set of $S-1$ thresholds parameters $\left(\widehat{\tau}_{i s s^{\prime}}\right)$ 
for each state $s$, that delineates the regions of switching. For example, consider a three-state model where customer $i$ is in state 2 in period $t-1$. If $u_{i t} \leq \widehat{\tau}_{i 21}$, customer $i$ would transition to state 1 . If $\widehat{\tau}_{i 21} \leq u_{i t} \leq \widehat{\tau}_{i 22}$, customer $i$ would stay in state 2 . And if $u_{i t} \geq \widehat{\tau}_{i 22}$, then customer $i$ would transition to state 3 .

More generally, the transition probabilities in Equation (2) are given by:

$$
\begin{aligned}
q_{i s 1 t} & =\frac{\exp \left(\widehat{\tau}_{i s 1}-\boldsymbol{\rho}_{i s}^{\prime} \cdot \mathbf{z}_{i t-1}\right)}{1+\exp \left(\widehat{\tau}_{i s 1}-\boldsymbol{\rho}_{i s}^{\prime} \cdot \mathbf{z}_{i t-1}\right)}, \\
q_{i s 2 t} & =\frac{\exp \left(\widehat{\tau}_{i s 2}-\boldsymbol{\rho}_{i s}^{\prime} \cdot \mathbf{z}_{i t-1}\right)}{1+\exp \left(\widehat{\tau}_{i s 2}-\boldsymbol{\rho}_{i s}^{\prime} \cdot \mathbf{z}_{i t-1}\right)}-\frac{\exp \left(\widehat{\tau}_{i s 1}-\boldsymbol{\rho}_{i s}^{\prime} \cdot \mathbf{z}_{i t-1}\right)}{1+\exp \left(\widehat{\tau}_{i s 1}-\boldsymbol{\rho}_{i s}^{\prime} \cdot \mathbf{z}_{i t-1}\right)}, \\
& \vdots \\
q_{i s S t} & =1-\frac{\exp \left(\widehat{\tau}_{i s S-1}-\boldsymbol{\rho}_{i s}^{\prime} \cdot \mathbf{z}_{i t-1}\right)}{1+\exp \left(\widehat{\tau}_{i s S-1}-\boldsymbol{\rho}_{i s}^{\prime} \cdot \mathbf{z}_{i t-1}\right)} .
\end{aligned}
$$

Equation (3) specifies how previous marketing actions and customer characteristics $\left(\mathbf{z}_{i t-1}\right)$ affect the transition probabilities. To ensure a proper ordering of the buying-behavior states, we impose a non-decreasing order for the thresholds $\left(\widehat{\tau}_{i s 1} \leq \widehat{\tau}_{i s 2} \leq \ldots \leq \widehat{\tau}_{i s S-1}\right)$ by setting $\widehat{\tau}_{i s 1}=\tau_{i s 1} ; \widehat{\tau}_{i s s^{\prime}}=\widehat{\tau}_{i s s^{\prime}-1}+\exp \left(\tau_{i s s^{\prime}}\right) \quad \forall i, s$, and $s^{\prime}=2, \ldots, S-1$.

3.1.3. Conditional Choice Probabilities. If the buying-behavior states are observed, one could directly estimate the Markov model defined by the transition matrix. Since the states are not observed, we propose the use of a HMM to uncover the buying-behavior states from the observed buying behavior. The HMM describes a stochastic process that is not directly observable (the latent state membership), but can be inferred through another stochastic process that relates the set of observations to the HMM latent state membership. Such observations correspond to the customer's decisions over time. We model the customer's decisions conditional on the customer being in a particular state and the marketing interventions as: ${ }^{1}$

$$
P_{i s t}=P\left(Y_{i t}=y_{i t} \mid X_{i t}=s, \mathbf{z}_{i t}\right),
$$

where we allow the marketing actions in period $t\left(\mathbf{z}_{i t}\right)$ to affect the customer's decision $\left(Y_{i t}\right)$. Following standard notation in HMM models, we write the vector of state dependent probabilities

\footnotetext{
${ }^{1}$ For continuous $Y_{i t}, P_{i s t}$ corresponds to the pdf $f\left(y_{i t} \mid X_{i t}=s, \mathbf{z}_{i t}\right)$.
} 
as a diagonal matrix $\left(\mathbf{M}_{i t}\right)$ :

$$
\mathbf{M}_{i t}=\left(\begin{array}{cccc}
P_{i 1 t} & 0 & \cdots & 0 \\
\vdots & \ddots & & \vdots \\
0 & \cdots & 0 & P_{i S t}
\end{array}\right)
$$

3.1.4. The Likelihood of an Observed Sequence of Choices. Given the Markovian structure of the model, the likelihood of observing a choice at time $t$ is dependent on all choices made in the past. Therefore, we write the joint likelihood of observing a sequence of $T$ decisions made by customer $i$ as follows:

$$
\begin{aligned}
& L_{i T}=P\left(Y_{i 1}, \ldots, Y_{i t}, \ldots, Y_{i T}\right)= \\
& \sum_{X_{i 1}=1}^{S} \cdots \sum_{X_{i T}=1}^{S} P\left(Y_{i 1}, \ldots, Y_{i T} \mid X_{i 1}, \ldots, X_{i T}\right) P\left(X_{i 1}, \ldots, X_{i T}\right)= \\
& \sum_{X_{i 1}=1}^{S} \cdots \sum_{X_{i T}=1}^{S} P\left(Y_{i 1} \mid X_{i 1}\right) \cdots P\left(Y_{i T} \mid X_{i T}\right) P\left(X_{i 1}\right) P\left(X_{i 2} \mid X_{i 1}\right) \cdots P\left(X_{i T} \mid X_{i T-1}\right)= \\
& \sum_{X_{i 1}=1}^{S} \cdots \sum_{X_{i T}=1}^{S} P\left(X_{i 1}\right) P\left(Y_{i 1} \mid X_{i 1}\right) P\left(X_{i 2} \mid X_{i 1}\right) P\left(Y_{i 2} \mid X_{i 2}\right) \cdots P\left(X_{i T} \mid X_{i T-1}\right) P\left(Y_{i T} \mid X_{i T}\right) .
\end{aligned}
$$

The population likelihood for a sample of $N$ random customers is given by $L=\prod_{i=i}^{N} L_{i T}$. McDonald and Zucchini (1997) show that the above likelihood expression can be succinctly written as:

$$
L=\prod_{i=1}^{N} \boldsymbol{\pi}_{i}^{\prime} \mathbf{M}_{i 1} \prod_{t=2}^{T} \mathbf{Q}_{i t} \mathbf{M}_{i t} \mathbf{1}
$$

where 1 is a Sx1 vector of ones.

3.1.5. Recovering State Membership. Our HMM approach allows us to recover the customers' membership to any state at any given time period. We compute the probabilistic membership using the filtering approach (McDonald and Zucchini 1997), which uses the information known up to period $t$ to recover the customer's membership at period $t$. The filtering probability that customer $i$ is in state $s$ at time $t$, conditioned on the customer's decisions up to period $t$, is given by:

$$
P\left(X_{i t}=s \mid Y_{i 1}, \ldots, Y_{i t}\right)=\boldsymbol{\pi}_{i} \mathbf{M}_{i 1} \prod_{t=2}^{T} \mathbf{Q}_{i t} \mathbf{m}_{i t}^{s} / L_{i t}
$$


where $\mathbf{m}_{i t}^{s}$ is the $s^{t h}$ column of the matrix $\mathbf{M}_{i t}$ and $L_{i t}$ is the likelihood of the observed sequence of customer $i$ 's decisions up to time $t$ from Equation (6).

\subsection{The Short- and Long-Term Effects of Marketing Activities}

Marketing mix interventions can have an immediate impact on the customer's behavior (e.g., product purchase due to sales promotion) with short-term implications and/or produce a change in the customer's buying-behavior state, which may have long-term implications (e.g., being upgraded to a higher tier in the loyalty programs).

Our model allows marketing interventions to have both short- and long-term effects and disentangles both effects in a very natural way. To capture the short-term effect, marketing actions are included in the state-dependent decision ( $\mathbf{z}_{i t}$ in Equation (4)). Behaviorally, this means that conditional on the customer's current buying-behavior state, marketing interventions may have an immediate effect on the likelihood and/or the quantity purchased. To capture the long-term effect, marketing interventions are included in the transition probabilities between buying-behavior states $\left(\mathbf{z}_{i t-1}\right.$ in Equation (2)). ${ }^{2}$ Behaviorally, this means that marketing interventions can move a customer from one buying-behavior state to another, possibly more favorable, state. As illustrated in the previous examples, this regime shift may have long-term impact on the customer's decisions depending upon the stickiness of the new state and the nature of the transitions from it. The set of marketing interventions $\mathbf{z}_{i t}$ to be included in the transition matrix (Equation (2)) or in the conditional choice component (Equation (4)) may be different. The location of the marketing interventions in our model can be determined a priori based on the researcher's hypotheses or managerial beliefs, or tested statistically following model selection criteria. In this paper, we locate the marketing actions in both the transition matrix $\mathbf{Q}_{i t}$ and in the conditional choice matrix $\mathbf{M}_{i t}$ to empirically assess the degree of shortand long-term impact of each marketing activity. ${ }^{3}$

\subsection{Optimal Marketing Mix Allocation}

In this section, we describe a dynamic programming (DP) optimization procedure used to optimally allocate and target marketing resources for long-term profitability.

\footnotetext{
${ }^{2}$ We include $\mathbf{z}_{i t-1}$ in the transition matrix to ensure precedence of $\mathbf{z}_{i t-1}$ to the customer's transition and the customer decision in period $t$. In situations where the customer is exposed to the marketing actions prior to making a decision in the same period (e.g., scanner panel data), one could replace $\mathbf{z}_{i t-1}$ with $\mathbf{z}_{i t}$.

${ }^{3}$ We have conducted a series of simulations to confirm the empirical identification of the short- and long-term effects. Details can be obtained from the authors.
} 
One aspect of the HMM that makes the dynamic optimization difficult is that the firm has uncertainty regarding the customers' state at any period $t$, and how the customers will evolve over time through the buying-behavior states. In other words, the state variable $X_{i t}$ is only probabilistically observed. Most of the DP applications in marketing utilize observed state variables such as past purchases (e.g., Bitran and Mondschein 1996; Simester et al. 2006). To address this complexity, we define the DP states using a partially observed Markov decision process (POMDP). A POMDP is a sequential decision problem, pertaining to a dynamic setting, where the information concerning the state of the system is incomplete (see e.g., Aviv and Pazgal 2005; Lovejoy 1991; Monahan 1982). In our case, the states of the DP problem are defined by the firm's beliefs about customers' state membership. Our approach, however, differs from other POMDPs described in the literature in that we assume the firm wants to predict the impact of the marketing interventions for an infinite horizon, not just the next period. This assumption has implications for how the firm updates its beliefs, as we describe below.

We define $b_{i t}$ as the firm's belief about the probability that customer $i$ is in state $s$ at time t. After observing the customer's decision and its own marketing intervention decision $\left(\mathbf{z}_{i t}\right)$, the firm can update its beliefs in a Bayesian manner. Specifically, using the Bayes' rule and the transition probability estimates $\left(q_{i s^{\prime} s t}\right)$ from Equations (2) and (3), the firm's beliefs about the customer's state can be updated from period $t$ to $t+1$ as:

$$
b_{i t+1}\left(s \mid \mathbf{B}_{i t}, \mathbf{z}_{i t-1}\right)=\frac{\sum_{s^{\prime}=1}^{S} b_{i t}\left(s^{\prime}\right) q_{i s^{\prime} s t}}{\sum_{s^{\prime}=1}^{S} \sum_{l=1}^{S} b_{i t}\left(s^{\prime}\right) q_{i s^{\prime} l t}},
$$

where $\sum_{s=1}^{S} b_{i t}(s)=1$. By defining $\mathbf{B}_{i t}=\left[b_{i t}(1) \cdots b_{i t}(S)\right]$, Equation (9) can be succinctly written as follows:

$$
\mathbf{B}_{i t+1}=\mathbf{B}_{i t} \mathbf{Q}_{i t}
$$

The updating process takes into account the firm's beliefs about the customer's state and its marketing mix decisions in the previous period. ${ }^{4}$ Therefore, $\mathbf{B}_{i t}$ summarizes all the information available for making decisions at time $t$. Furthermore, for any fixed sequence of marketing actions $\mathbf{z}_{i 1}, \ldots, \mathbf{z}_{i t}$ the sequence of probabilities $\mathbf{B}_{i t}$ is a Markov process.

\footnotetext{
${ }^{4}$ We assume that $b_{i t}(s)$ reflects the firm's beliefs that customer $i$ is in state $s$ at the beginning of period $t$, before either the firm has implemented any marketing activity or the customer has made any decision.
} 
We model the firm's decision process as a dynamic programming problem under customers' state uncertainty. The objective of the firm is to determine, for each period, the best marketing intervention, so as to maximize the sum of discounted expected future profits $R_{i t}$, over an infinite planning horizon. That is,

$$
\max _{\mathbf{z}_{i t}} E\left\{\sum_{\tau=t}^{\infty} \delta^{\tau-t} R_{i \tau}\right\},
$$

where, $\delta(0 \leq \delta \leq 1)$ is the discount rate, $E\left[R_{i t}\right]=\sum_{s=1}^{S} b_{i t}(s) r_{i s t}$, and $r_{i s t}$ is the profit earned by the firm during period $t$ if customer $i$ is in state $s$ and given marketing intervention $\mathbf{z}_{i t}$.

Note that customer $i$ 's HMM (defined in Section 3.1) enters into the firm's expected profits in two places: the transition matrix $\left(\mathbf{Q}_{i t}\right)$ affects the firm's beliefs about the state membership of customer $i\left(\mathbf{B}_{i t}\right)$; and the conditional choice behavior matrix $\left(\mathbf{M}_{i t}\right)$ affects the state dependent profits $\left(r_{i s t}\right)$.

The firm's optimal scheduling of marketing interventions is the solution to the dynamic program from that time forward. Following the Bellman (1957) equation, the DP problem can be written as:

$$
\begin{aligned}
\sum_{i=1}^{N} V_{i}^{*}\left(\mathbf{B}_{i t}\right) & =\sum_{i=1}^{N} \max _{\mathbf{z}_{i t}} E\left\{\sum_{\tau=t}^{\infty} \delta^{\tau-t} R_{i \tau}\right\} \\
& =\sum_{i=1}^{N} \max _{\mathbf{z}_{i t}}\left\{\sum_{s=1}^{S} b_{i t}(s) \cdot r_{i s t}+\delta\left[V_{i}^{*}\left(\mathbf{B}_{i t+1}\right)\right]\right\},
\end{aligned}
$$

subject to

$$
\begin{aligned}
& \mathbf{B}_{i t+1}=\mathbf{B}_{i t} \mathbf{Q}_{i t} \quad \forall i, t \quad \text { (update beliefs) } \\
& \sum_{i=1}^{N} \mathbf{z}_{i t} \leq \mathbf{A}_{t} \quad \forall t \quad \text { (marketing budget) } \\
& \mathbf{z}_{i t} \in \mathbf{D} \quad \forall i, t \quad \text { (marketing actions space), }
\end{aligned}
$$

where $V_{i}^{*}\left(\mathbf{B}_{i t}\right)$ denotes the maximum discounted expected profits that can be obtained for customer $i$ given the current beliefs $\left(\mathbf{B}_{i t}\right), \mathbf{A}_{t}=\left\{a_{t j}\right\}_{j=1}^{J}$ corresponds to the marketing budget available at time $t$ described in units of marketing activity $j, \mathbf{D}$ is the set of possible actions, and $N$ is the total number of customers.

The exact solution to this DP problem involves complex computations and it is unsolvable using direct dynamic optimization techniques because of the continuous nature of the statespace of beliefs. To solve the infinite-horizon continuous-state DP problem, we combine two 
approximation approaches: (i) value function iteration, and (ii) value function interpolation. In the value function iteration procedure, we first assume that there is a terminal period $T$ at which the future reward is exactly zero for all firm's beliefs about customers. Then, at $t=T-1$, Equation (12) takes a simple form as the second term in the equation disappears. This permits solving the DP problem by backward induction. We do so for a sufficiently large number of time periods so that the value functions become stable, meaning that they cease to change significantly as we move further back (see e.g. Erdem et al. 2003). We use the value function interpolation procedure to approximate the continuous state-space of beliefs. This approach involves solving for the individual customer value function on a grid of state points and then interpolating for other points in the state-space (see Keane and Wolpin 1994 for details).

In order to allocate marketing resources for a given budget, we solve the DP problem using a backward induction procedure simultaneously for all customers. To find the optimal budget and allocate the marketing activities across customers, we solve the same problem described above, but without the budget constraint in Equation (12). Without the budget constraint, the problem can be solved independently for each customer using the value iteration/interpolation approach described above. ${ }^{5}$

As we demonstrate in the empirical application, the solution to the dynamic allocation problem provides important managerial implications for the firm regarding the optimal scheduling and allocation of the marketing interventions for each customer over time. Furthermore, by considering the enduring effects of marketing actions, firms can efficiently maximize long-run profitability.

\section{Empirical Application}

In this section, we describe an application of the proposed model in the context of physicians' prescription behavior over two years following the introduction of a new drug. Our objective is to manage the physician base for long-run profitability through an efficient marketing mix allocation. We first estimate the proposed HMM and asses the impact and duration of the effects of the marketing efforts. We then use these estimates to solve the dynamic optimization problem described in the previous section.

\footnotetext{
${ }^{5}$ Details of the approximation techniques and solution to the DP problems can be found in Appendix A.
} 


\subsection{Pharmaceutical Marketing}

There are several reasons for our choice of pharmaceutical marketing as an empirical application for the proposed model. First, pharmaceutical marketing is one of the most prevalent areas of marketing research. This stems from the importance of the industry, the amount of money spent on pharmaceutical marketing activities, and the availability of high quality data. It is estimated that there are approximately 100,000 sales representatives in the United States pursuing some 830,000 physicians. ${ }^{6}$ Second, previous research suggests that physicians may indeed exhibit dynamic prescription behavior. For a new drug, it has been suggested that such dynamics may result from physicians' learning (Janakiraman et al. 2005; Mukhertji et al. 2004; Narayanan and Manchanda 2005). Third, research has shown that pharmaceutical marketing actions can have both short- and long-term effects (Gönül et al. 2001; Manchanda and Chintagunta 2004; Mizik and Jacobson 2004; Narayanan et al. 2005).

Since pharmaceutical marketing actions are often individually targeted, the problem of allocating marketing resources becomes difficult; especially in light of physicians' heterogeneity, dynamics in prescription behavior, and the possibility of enduring effects of marketing actions. Previous research suggests that pharmaceutical companies do not allocate their marketing budgets optimally across physicians (Chintagunta and Vilcassim 1994; Manchanda and Chintagunta 2004; Narayanan et al. 2005). However, to the best of our knowledge, none of the studies mentioned above have integrated physicians' dynamics and the long-term effects of marketing actions to determine the optimal allocation of marketing resources. This research offers a first step in providing marketing managers with models for managing physicians through an efficient and dynamic allocation of marketing resources.

\subsection{Data}

Our data set comprises physician-level new prescriptions and marketing mix effort over a 24 month period post-launch of a new drug used to treat a medical condition in postmenopausal women. These data are compiled from internal company records and pharmacy audits. ${ }^{7}$ The data set contains, for each physician and each month, the number of new prescriptions written for both the new drug and the category, and the number of details and samples received for the

\footnotetext{
${ }^{6}$ Robert Ebisch. 2005. Prescriptions for change. Teradata Magazine, March.

${ }^{7}$ For confidentiality reasons, we cannot reveal the name of the new drug or the company that provided us with the data.
} 
new drug. Detailing corresponds to phone calls or face-to-face meetings where pharmaceutical representatives present information about the drugs to physicians. Sampling corresponds to the practice of giving free drug samples to physicians by pharmaceutical representatives. ${ }^{8}$ Our sample consists of 300 physicians who have received at least one detail and one sample during the first 12 months of the data.

Table 1 presents descriptive statistics of the data. On average, a physician writes 22.5 new prescriptions in the category per month, 1.63 of which correspond to the new drug. Additionally, each physician receives 1.94 details and 3.71 samples of the new drug per month. Furthermore, the range statistics in Table 1 demonstrates a high degree of physician heterogeneity in prescription behavior as well as in the number of details and samples received.

Table 1: Summary of descriptive statistics ${ }^{*}$ (per physician)

\begin{tabular}{lrrrrr}
\hline & Mean & Std. dev. & Lower $10 \%$ & Median & Upper 90\% \\
\hline New drug prescriptions & 1.62 & 1.35 & 0.54 & 1.33 & 3.21 \\
Details & 1.94 & 0.74 & 1.10 & 1.83 & 2.94 \\
Samples & 3.71 & 2.63 & 1.00 & 2.92 & 7.48 \\
Category prescriptions & 22.50 & 13.05 & 10.10 & 18.85 & 37.79 \\
New drug share & 0.079 & 0.058 & 0.026 & 0.065 & 0.143 \\
\hline
\end{tabular}

* Average monthly values computed for each physician across the sample of 300 physicians.

Figure 2 shows the monthly evolution of the total volume of new drug prescriptions, details, samples, and share of prescriptions for the 24-month span of our data. The figure suggests an increasing trend in the level of prescriptions of the new drug but relatively stable detailing and sampling activities by the firm. Additionally, Figure 2 shows that the share of the new drug steadily increases from almost $0 \%$ in the first month to about $10 \%$ share-of-prescriptions in the last month, following closely the increase in prescriptions of the new drug. Thus, the increase in the volume of prescriptions for the new drug cannot be attributed solely to category expansion. Since the new drug reaches only $10 \%$ share by month 24 , it seems that demand for the new drug has not reached saturation by the end of our observation period..$^{9}$

Several questions may arise from Figure 2: (i) How did the marketing actions (detailing and sampling) influence physicians' prescribing behavior? (ii) Do these marketing activities have primarily a short-term impact or an enduring effect? (iii) Could the firm have implemented a better targeting policy? We address these and other questions in the following sections.

\footnotetext{
${ }^{8}$ In this application, a drug sample corresponds to the equivalent of one-month prescription.

${ }^{9}$ Since the incidence of the medical problem is not affected by seasonal variables, any dynamics observed in the data should not be due to seasonality effects.
} 
Figure 2: Total number of new drug prescriptions, details, samples, and share per month.

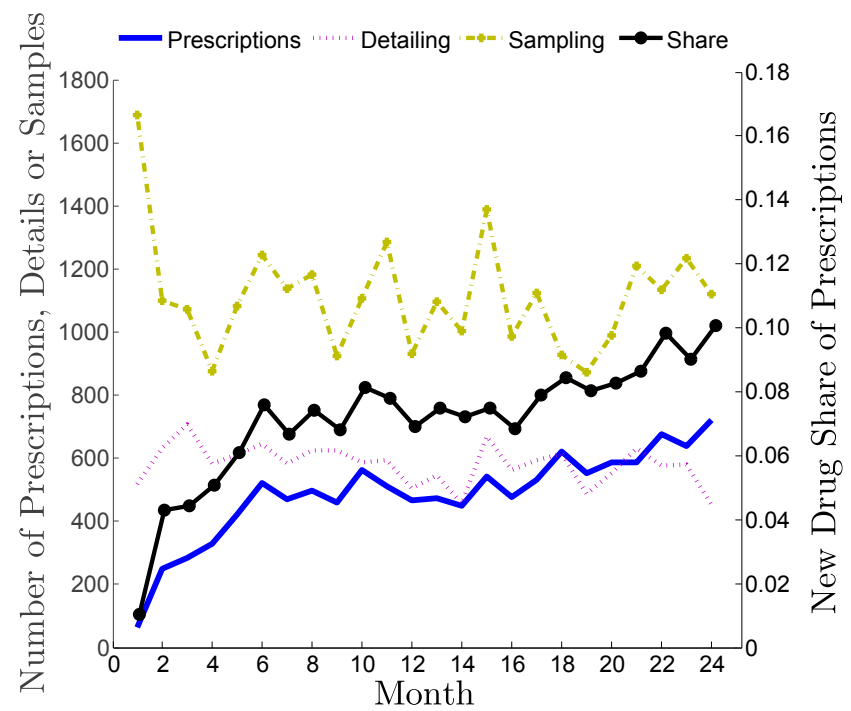

\subsection{Current Targeting Policy}

Before we describe the implementation of our model to the direct-to-physician data, it is instructive to analyze first the targeting and resource allocation policy employed by the firm. A conversation with the data provider revealed that the firm targets individual physicians based on the level of prescriptions they have written in the category, such that high volume category prescribers are targeted with more details and samples than low volume category prescribers. ${ }^{10}$ This targeting policy is commonly used in the pharmaceutical industry. We explore, how this policy was implemented in practice. That is, whether physicians with higher category prescription volume effectively received more details and samples than other physicians.

Following industry practice, we divide the physicians base into 10 deciles based on their category volume of prescriptions in the past three months. Then, we create three groups, where the first group corresponds to deciles 1 to 4, the second group corresponds to deciles 5 to 7 , and the third group corresponds to deciles 8 to 10. Table 2 shows that physicians in the higher deciles received more details and samples than physicians in the lower deciles $(p<0.01$, contrast test of linear increasing trend). Thus, we find statistical evidence that detailing and sampling were targeted according to category prescription deciles.

\footnotetext{
${ }^{10}$ Some pharmaceutical companies may use additional information such as: profitability of a prescription, accessibility of the physician, tendency of the physician to use the pharmaceutical company's drugs, the tendency of the physician to use a wide palette of drugs, and the influence that physicians have on their colleagues. These data, however, are usually not available.
} 
Table 2: Current targeting policy: Quarterly detailing and sampling by decile

\begin{tabular}{clccc}
\hline & & \multicolumn{3}{c}{ Deciles } \\
\cline { 3 - 5 } & & $1-4$ & $5-7$ & $8-10$ \\
\hline \multirow{5}{*}{ Details } & Mean & 5.57 & 6.00 & 5.80 \\
& Std. dev. & $(0.31)$ & $(0.22)$ & $(0.14)$ \\
& Mean & 8.65 & 11.95 & 13.04 \\
& Std. dev. & $(1.19)$ & $(0.78)$ & $(1.72)$ \\
\hline
\end{tabular}

Therefore, there seems to be endogeneity in the sampling and detailing targeting policy at the category level (Manchanda et al. 2004). However, our model is specified at the drug rather than the category level. Thus, detailing and sampling targeting policy would suffer from endogeneity only if the total category volume of prescriptions and the share of the new drug prescriptions are correlated. We explore this issue by replicating the above analysis using the share of prescriptions as the dependent variable, instead of detailing or sampling. We find low linear correlation between the total volume of prescriptions in the category and the share of prescriptions $(r=-0.096)$. Further, the average shares of prescriptions of each group are not statistically different across groups (equal mean test cannot be rejected; $p>0.1$ ). Thus, we find no evidence of endogeneity at the drug level, possibly since we are dealing with a new drug. We further explore possible endogeneity by running linear regressions of the marketing activities applied in periods 21-24, as functions of the responsiveness to marketing activities. We obtain the physician-level responsiveness to marketing actions by estimating a heterogeneous binomial model of number of new drug prescriptions, which corresponds to a one state HMM (see next section for the specification of the model). We did not find a significant correlation between responsiveness to detailing and sampling and the number of details or samples offered to physicians $(p>0.1)$.

In summary, we find no evidence in our data that marketing mix allocation is based on physicians' responsiveness to these activities or the new drug prescription. In contrast, there is evidence that marketing resources are allocated based on the total number of category prescriptions written by physicians. Hence, endogeneity is not a serious concern in estimating our model, which is based on the prescriptions of the new drug. 


\subsection{Model Specification}

To parsimoniously model the physician prescription behavior and the effects of detailing and sampling, we make a few modifications to each one of the three components of the general HMM described in Section 3.1.

1. Initial state-membership probabilities. Since this is a new drug, we assume that all physicians start at state 1 , which corresponds to the lowest prescription-behavior state, in the first month. ${ }^{11}$ Thus,

$$
\boldsymbol{\pi}_{i}=\left[\pi_{i 1}, \pi_{i 2}, \ldots, \pi_{i S}\right]=[1,0, \ldots, 0] .
$$

2. Markov chain transition matrices. Our data set includes two marketing actions that may have long-term effects on the physicians' prescription behavior and should be included in the transition matrix: detailing and sampling. Accordingly, the vector of marketing actions in Equations (2) and (3) includes:

$$
\mathbf{z}_{i t}=\left[f\left(\text { Detailing }_{i t}\right), f\left(\text { Sampling }_{i t}\right)\right],
$$

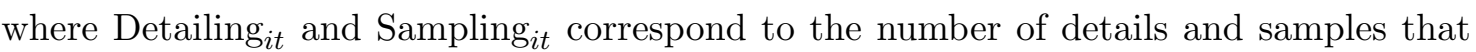
physician $i$ receives in month $t$, and $f(x)=\frac{\ln (x+1)-\mu}{\sigma}$, where $\mu=\operatorname{mean}(\ln (x+1))$, and $\sigma=\operatorname{std}(\ln (x+1))$. We $\log$ transform detailing and sampling to capture the potentially diminishing returns of their effectiveness (Gönül et al. 2001; Manchanda and Chintagunta 2004). We normalize these variables to ensure a proper identification of the prescriptionbehavior states (as we describe later).

3. Conditional choice behavior. This component of the HMM captures the physicians' prescription behavior conditional on their state. We model the prescription behavior $Y_{i t}$ as the number of prescriptions written by physician $i$ in month $t$. To account for category demand, let $W_{i t}$ be the total number of new prescriptions in the category written by physician $i$ in month $t$. Then, the number of prescriptions of the new drug $Y_{i t}$ has a binomial distribution with parameters $p_{i s t}$ and $W_{i t}$, where $p_{i s t}$ is the probability of physician $i$ prescribing the new drug in month $t$. That is,

$$
P_{i s t}\left(Y_{i t}=y_{i t} \mid X_{i t}=s, \mathbf{z}_{i t}\right)=\left(\begin{array}{c}
W_{i t} \\
y_{i t}
\end{array}\right) p_{i s t}^{y_{i t}}\left(1-p_{i s t}\right)^{W_{i t}-y_{i t}} .
$$

\footnotetext{
${ }^{11}$ The more general specification of estimating the vector $\boldsymbol{\pi}_{i}$ provides no significant improvement in fit.
} 
To capture the short-term impact of marketing actions on share-of-prescriptions, we define:

$$
p_{i s t}=\frac{\exp \left(\widehat{\alpha}_{s}^{0}+\boldsymbol{\alpha}_{i s}^{\prime} \mathbf{z}_{i t}\right)}{1+\exp \left(\widehat{\alpha}_{s}^{0}+\boldsymbol{\alpha}_{i s}^{\prime} \mathbf{z}_{i t}\right)}
$$

where $\widehat{\alpha}_{s}^{0}$ is the intrinsic probability of prescribing given state $s$, and $\mathbf{z}_{i t}$ includes the transformed Detailing $i t$ and Sampling $i t$ variables in Equation (14). To ensure the identification of the states we impose that the choice probabilities in the binomial model are non-decreasing in the behavioral states. We impose this restriction at the mean of the vector of covariates, $\mathbf{z}_{i t}$, such that $\widehat{\alpha}_{1}^{0} \leq \cdots \leq \widehat{\alpha}_{S}^{0}$ is imposed by $\widehat{\alpha}_{1}^{0}=\alpha_{1}^{0}$; $\widehat{\alpha}_{s}^{0}=$ $\widehat{\alpha}_{s-1}^{0}+\exp \left(\alpha_{s}^{0}\right) \forall s=2, \ldots, S$.

There are several advantages for using the binomial distribution in the current application. First, since we do not model patients' demand explicitly, by accounting for category prescriptions we control for variation in patients' demand in the entire category. Second, category prescriptions help to control for any seasonal or time-specific effects that may affect the market or the specific physician. Third, the binomial distribution can easily handle extreme values of share-of-prescriptions observed in our data (0 and 1).

Plugging Equations (13), (15), and (16) into Equation (7) produces the likelihood of observing a sequence of prescriptions.

\subsection{Model Estimation}

In this section we briefly discuss the procedure we use to estimate our model. To ensure that cross individual heterogeneity is distinguished from time dynamics we specify the HMM parameters at the individual level. ${ }^{12}$ We estimate the transition matrix parameters and the state dependent choice parameters described in Equations (2)-(4) and (13)-(16) using the joint likelihood function in Equation (7). We define $\boldsymbol{\Phi}=\left\{\alpha_{s}^{0}\right\}_{s=1}^{S}$ as the set of fixed-effect parameters and $\boldsymbol{\theta}_{i}=\left\{\tau_{i s 1}, \ldots, \tau_{i s S-1}, \boldsymbol{\rho}_{i s}, \boldsymbol{\alpha}_{i s}\right\}_{s=1}^{S}$ as the set of random-effect parameters. We estimate the random- and fixed-effect HMM parameters using a hierarchical Bayes Markov Chain Monte Carlo (MCMC) procedure (Rossi et al. 2005).

In the hierarchical Bayes procedure, we recursively draw from the conditional distribution of each parameter. Given that the conditional posterior distributions of $\boldsymbol{\theta}_{i}$ and $\boldsymbol{\Phi}$ do not have

\footnotetext{
${ }^{12}$ For practical purposes we assume that the intrinsic conditional probabilities of prescribing $\alpha_{s}^{0}$ in Equation (16) do not vary across physicians. This helps us to interpret and compare physicians' behavior across states in a normalized way.
} 
a closed form, we employ a Gibbs sampler and a random-walk Metropolis-Hastings procedure to draw candidates and obtain the conditional distribution for each of the parameters. Unlike HMM Bayesian estimation methods that augment the latent state membership (Djurić and Chun 2002; Kim and Nelson 1999; Moon et al. 2007; Scott 2002), we follow Netzer et al. (2007) and use the Bayesian approach to estimate directly the likelihood function in Equation (7) with random- and fixed-effect parameters. Due to the intrinsic autocorrelation of the HMM, which leads to autocorrelation between successive draws of the MCMC chain, we use the adaptive procedure proposed by Atchadé (2006) which improves convergence and mixing properties. See Appendix B for a further description of the MCMC estimation procedure and the prior and posterior distributions of the HMM.

We use the first 20 months of data to estimate the model and the last four months for validation purposes. We ran the hierarchical Bayes estimation for 300,000 iterations. The first 200,000 iterations were used as a "burn-in" period and the last 100,000 iterations were used to estimate the conditional posterior distributions. Convergence was assessed by running multiple parallel chains following Gelman and Rubin's criterion (Gelman and Rubin 1992).

\subsection{Results and Marketing Implications}

4.6.1. Model Selection. The number of states could be estimated from data or specified a priori based on theoretical grounds. We take the former approach and treat the number of states as a parameter to be estimated to optimize goodness of fit criteria. Specifically, to choose the number of states (S) we used the marginal log density (MLL), the deviance information criterion (DIC), the log Bayes factor (Log BF), and the validation log-likelihood. All these measures are calculated from the output of the Metropolis-Hastings sampler. The MLL is calculated using the harmonic mean of the individual likelihoods across iterations (Newton and Raftery 1994). A one-state model corresponds to a binomial model without dynamics, which is characterized by equations (15) and (16).

The results in Table 3 suggest selecting a three-state model. This model maximizes the marginal log density, shows a favorable log Bayes factor in comparison to the models with two and four states, minimizes the DIC value, and shows the best predictive fit (highest validation log-likelihood) for the validation periods (periods 21-24). ${ }^{13}$

\footnotetext{
${ }^{13}$ We also tested a four-state model with an absorbing no prescription ("defected") state. The fit criteria for this model are: $\mathrm{MLL}=-8517$, DIC $/ 2=8840$, Validation log-likelihood $=-2195$. Therefore, a model where physicians can move to a "defected" state is rejected in favor of a model with three states. The constrained model is also rejected
} 
Table 3: Selecting the number of states

\begin{tabular}{crrrr}
\hline & & & & $\begin{array}{r}\text { Validation } \\
\text { States }\end{array}$ \\
\hline 1 & -10854 & 11001 & & -2842 \\
2 & -9037 & 9207 & 1817 & -2240 \\
$\mathbf{3}$ & $\mathbf{- 8 4 6 8}$ & $\mathbf{8 7 9 1}$ & $\mathbf{5 6 8}$ & $\mathbf{- 2 1 7 1}$ \\
4 & -8489 & 8859 & -21 & -2179 \\
\hline
\end{tabular}

4.6.2. Predictive Validity. In this section we compare the predictive validity of the selected three-state HMM relative to four benchmark models: two nested versions of the HMM, a latent class model, and an RFM model. The latter two are commonly used in the literature to capture heterogeneity and dynamics in buying behavior. We use the first 20 periods to calibrate the model and the last four periods for validation purposes. For all the models, we assume that prescriptions follow a binomial distribution and use a MCMC Metropolis-Hastings approach to estimate the model parameters.

Nested HMM models. In addition to our full three-state HMM model (Full HMM-3), we estimate two nested versions of the model. The first is a fixed-parameter three-state HMM, where the parameters do not vary across physicians (Fixed-Parameter HMM-3). This model allows us to assess the magnitude of heterogeneity among physicians in the sample. The second is a three-state HMM with a stationary transition matrix and the marketing activities included in the conditional choice component (M), allowing for only short-term effect of marketing actions (Stationary HMM-3). This model allows us to assess the value of capturing the long-term effect of marketing actions.

Latent Class (LC). The latent class model of Kamakura and Russell (1989) captures heterogeneity in customer behavior through a set of latent segments. However, this model cannot captures dynamics since customers cannot transition among segments. Thus, it can be viewed as a special case of a HMM, where the transition matrix is constrained to be an identity matrix and the initial state or segment membership probabilities are estimated from the data. That is, an individual customer can be at any given state in the initial period, but once she is in a state, she stays in that absorbing state during the remaining periods. We estimate this model for three segments to emphasize the differences between a model that accounts for heterogeneity

against an unconstrained four-state model in terms of $\log \mathrm{BF}$ and validation log-likelihood. Note, however, that the DIC value is better for the constrained model than for the four-state HMM. 
only and a model that accounts for both heterogeneity and dynamics.

RFM model. One of the models most commonly used to capture dynamics and manage the firm's customer base is the recency, frequency and monetary value model (Bitran and Mondschein 1996, Colombo and Jiang 1999, Pfeifer and Carraway 2000). We construct the RFM variables as follows: recency corresponds to the number of months since the last prescription, frequency corresponds to the average incidence of prescription up to the current time period, and monetary value is captured by the monthly average quantity of prescriptions of the new drug up to the current time period. These variables are defined at the physician level and are updated every month. Additionally, we add the transformed detailing and sampling variables to account for the effect of marketing activities.

We use the cross-validation method to asses the predictive validity of the alternative models. In this method, the predictive distribution of the holdout observations is derived to examine whether the actual data points fall in regions of reasonably high density. If we partition the data as $y=\left[y_{\text {out }}, y_{-o u t}\right]$, where $y_{\text {out }}$ is the set of "holdout" observations to be removed, and $y_{-o u t}$ describes the "calibration" set of observations, the density of the posterior predictive distribution can be written as:

$$
p\left(y_{\text {out }} \mid y_{- \text {out }}\right)=\int_{\boldsymbol{\Theta}} p\left(y_{\text {out }} \mid \boldsymbol{\Theta}\right) p\left(\boldsymbol{\Theta} \mid y_{- \text {out }}\right) d \boldsymbol{\Theta}
$$

where $\boldsymbol{\Theta}$ is the vector of the model's parameters.

One way of assessing the predictive validity is to compute the conditional predictive ordinate (CPO) (Geisser 1993; Gelfand and Dey 1994; Manchanda and Chintagunta 2004). The logarithm of the CPO for a given model, or the validation log-likelihood, calculated via MCMC methods, is given by:

$$
\log [C P O]=\sum_{\text {out }=1}^{N_{o}} \log \left[p\left(y_{\text {out }} \mid y_{- \text {out }}\right)\right]=\sum_{\text {out }=1}^{N_{o}} \log \left(\frac{1}{N_{k}} \sum_{k=1}^{N_{k}} p\left(y_{\text {out }} \mid \mathbf{\Theta}^{k}\right)\right)
$$

where $N_{o}$ is the number of observations in the validation set, $N_{k}$ is the number of MCMC iterations, and $p\left(y_{\text {out }} \mid \mathbf{\Theta}^{k}\right)$ is the likelihood for the validation period.

Based on the validation log-likelihood and the RMSE criteria (see Table 4), the selected three-state HMM (Full HMM-3) predicted the holdout prescription data best. Moreover, the superior fit and prediction of the dynamic fixed-parameter HMM (Fixed-Parameter HMM- 
3) relative to the LC model suggests that the degree of dynamics in our data is substantial. Similarly, by incorporating the effect of detailing and sampling in both the transition and conditional choice matrices, we not only capture and disentangle the short- and long-term effects of the pharmaceutical marketing but also improve the representation and prediction of physicians' behavior.

Table 4: Predictive validity

\begin{tabular}{lrrr}
\hline & \multicolumn{3}{c}{ Validation } \\
Model & MLL & log-likelihood & RMSE \\
\hline Full HMM-3 & -8468 & -2171 & 0.075 \\
Stationary HMM-3 & -8597 & -2177 & 0.077 \\
Fixed-Parameter HMM-3 & -9334 & -2232 & 0.089 \\
RFM & -9084 & -2261 & 0.075 \\
Latent Class & -10495 & -2357 & 0.087 \\
\hline
\end{tabular}

4.6.3. The HMM's Parameter Estimates. We now discuss the parameter estimates for the selected three-states HMM. In Table 5 we report the posterior means and posterior standard deviations of the parameters, as well as the $95 \%$ heterogeneity intervals.

In what follows, we use the HMM parameter estimates in Table 5 to (i) interpret the three HMM states, (ii) investigate physicians' dynamics, (iii) disentangle the short- and long-term effects of marketing actions, and (iv) explore the degree of heterogeneity among physicians.

(i) Interpreting the States. To characterize the three states, we convert the intercept parameters $\left(\alpha_{1}^{0}, \alpha_{2}^{0}, \alpha_{3}^{0}\right)$ in Table 5 into prescription probabilities (i.e., share of prescriptions) conditional on being in each state (Equation (16)) with and without detailing and sampling. The results in Table 6 suggest that if physicians are in the first state, their probability of prescribing is very close to zero. Accordingly, we call this the "inactive" state. In the second state, physicians present a somewhat more favorable prescription behavior towards the new drug - representing a $6 \%$ of the total volume of new prescriptions. Thus, we call this the "infrequent" state. In the third state, physicians frequently prescribe the drug to their patients, with prescription share nearing $20 \%$. Consequently, we label this state as the "frequent" state. Note that even in the frequent state, the share-ofprescriptions reaches only $20 \%$. This is typical for a new drug, but it also suggests that, even at the frequent state, physicians do not "run out" of patients to prescribe the drug to. To further characterize the states, we focus on the physicians' dynamics among the states next. 
Table 5: Posterior means, ${ }^{a}$ standard deviations, and $95 \%$ heterogeneity intervals $^{b}$

\begin{tabular}{|c|c|c|c|c|c|}
\hline & Par. label & Mean & Std. dev. & $2.5 \%$ & $97.5 \%$ \\
\hline \multicolumn{6}{|l|}{ Transition matrix } \\
\hline \multicolumn{6}{|l|}{ Intercepts } \\
\hline Low threshold - state 1 & $\tau_{11}$ & 0.36 & 0.12 & -0.34 & 1.53 \\
\hline High threshold - state 1 & $\tau_{12}$ & 1.67 & 0.13 & 1.10 & 2.05 \\
\hline Low threshold - state 2 & $\tau_{21}$ & -1.80 & 0.14 & -2.79 & -0.72 \\
\hline High threshold - state 2 & $\tau_{22}$ & 1.53 & 0.11 & 0.52 & 2.31 \\
\hline Low threshold - state 3 & $\tau_{31}$ & -1.98 & 0.23 & -3.45 & -1.16 \\
\hline High threshold - state 3 & $\tau_{32}$ & 0.77 & 0.19 & 0.07 & 1.30 \\
\hline Marketing effects & & \multicolumn{4}{|c|}{ Long-term } \\
\hline Detailing - state 1 & $\rho_{1}^{d}$ & 0.31 & 0.12 & -0.36 & 0.88 \\
\hline Detailing - state 2 & $\rho_{2}^{d}$ & 0.02 & 0.15 & -0.56 & 0.47 \\
\hline Detailing - state 3 & $\rho_{3}^{d}$ & 0.02 & 0.17 & -0.64 & 0.44 \\
\hline Sampling - state 1 & $\rho_{1}^{s}$ & 0.18 & 0.11 & -0.43 & 0.78 \\
\hline Sampling - state 2 & $\rho_{2}^{s}$ & 0.21 & 0.13 & -0.26 & 0.82 \\
\hline Sampling - state 3 & $\rho_{3}^{s}$ & 0.28 & 0.17 & -0.19 & 0.58 \\
\hline \multicolumn{6}{|l|}{ Conditional choice } \\
\hline \multicolumn{6}{|l|}{ Intercepts } \\
\hline Intercept - state 1 & $\alpha_{1}^{0}$ & -4.98 & 0.11 & & \\
\hline Intercept - state 2 & $\alpha_{2}^{0}$ & 0.83 & 0.05 & & \\
\hline Intercept - state 3 & $\alpha_{3}^{0}$ & 0.19 & 0.04 & & \\
\hline Marketing effects & & \multicolumn{4}{|c|}{ Short-term } \\
\hline Detailing - state 1 & $\alpha_{1}^{d}$ & 0.27 & 0.11 & -0.72 & 1.05 \\
\hline Detailing - state 2 & $\alpha_{2}^{d}$ & 0.00 & 0.05 & -0.61 & 0.51 \\
\hline Detailing - state 3 & $\alpha_{3}^{d}$ & -0.04 & 0.09 & -0.42 & 0.46 \\
\hline Sampling - state 1 & $\alpha_{1}^{s}$ & 0.09 & 0.11 & -0.73 & 0.98 \\
\hline Sampling - state 2 & $\alpha_{2}^{s}$ & 0.06 & 0.05 & -0.43 & 0.60 \\
\hline Sampling - state 3 & $\alpha_{3}^{s}$ & 0.02 & 0.08 & -0.42 & 0.51 \\
\hline
\end{tabular}

Table 6: State-specific share of prescription estimates with and without sampling and detailing*

\begin{tabular}{lrrrr}
\hline States & & Intercept & Detailing & Sampling \\
\hline Inactive & $p_{1}$ & 0.004 & 0.007 & 0.004 \\
Infrequent & $p_{2}$ & 0.062 & 0.065 & 0.067 \\
Frequent & $p_{3}$ & 0.196 & 0.187 & 0.201 \\
\hline
\end{tabular}

\footnotetext{
${ }^{*}$ Values correspond to the logit probabilities of prescribing. Intercept column indicates the intrinsic share conditional on being in a given state when detailing and sampling are zero. The detailing and sampling columns indicate the shares conditional on receiving the average number of details or samples.
} 
(ii) Physicians Dynamics. For ease of interpretation, we convert the transition matrix parameters in Table 5 to probability transition matrices, with and without the effect of marketing activities. This allows us to assess the dynamics in prescription behavior as physicians transition among these states.

Table 7: Posterior mean of the transition matrix across physicians ${ }^{*}$

No marketing
activities
\begin{tabular}{|l|l|l|}
\hline 0.75 & 0.25 & 0.00 \\
\hline 0.17 & 0.78 & 0.05 \\
\hline 0.15 & 0.46 & 0.39 \\
\hline
\end{tabular}$\quad$\begin{tabular}{|l|l|l|}
\hline 0.62 & 0.38 & 0.00 \\
\hline 0.16 & 0.79 & 0.05 \\
\hline 0.15 & 0.45 & 0.40 \\
\hline
\end{tabular}

\footnotetext{
The detailing and sampling matrices are calculated assuming the firm
} allocates the average number of details and samples to the physicians.

Examining the left-hand side matrix in Table 7, which represents the mean transition matrix with no detailing or sampling, we observe a high degree of stickiness in the inactive and infrequent states. That is, physicians currently in these states are very likely to remain in the same state in the next period. In contrast, in the frequent state, physicians are more likely to drop to the infrequent state, than they are to stay in the frequent state. ${ }^{14}$ Thus, consistent with Janakiraman et al. (2005), who find a high degree of physician persistence, we find that physicians are reluctant to fully adopt the new drug as they stick to the inactive and infrequent states. A possible explanation for such persistency to the low prescription states may be learning and search cost minimization (Janakiraman et al. 2005).

Dynamics in state membership. An alternative way to asses physicians' dynamics is to calculate the aggregate state membership distribution over time. We use Equation (8) to asses the probability of state membership for each physician at each time period. We, then, assign physicians to the state with the highest membership probability following a "max probability rule".

Figure 3 shows the physicians' state membership evolution over time. Given that this is a new drug introduction, the entire physician base started in the inactive state. The majority of physicians quickly moved to the infrequent state and to a lesser extent to the frequent state. Moreover, it took approximately 10 months for the distribution of

\footnotetext{
${ }^{14}$ When physicians reach the inactive, infrequent, or frequent states, they stay on average $4,4.7$, and 1.6 periods, respectively. The number of periods in a state is computed by $\left(1-q_{s s}\right)^{-1}$, where $q_{s s}$ is the probability of staying in state $s$.
} 
Figure 3: Distribution of physicians' state membership over time

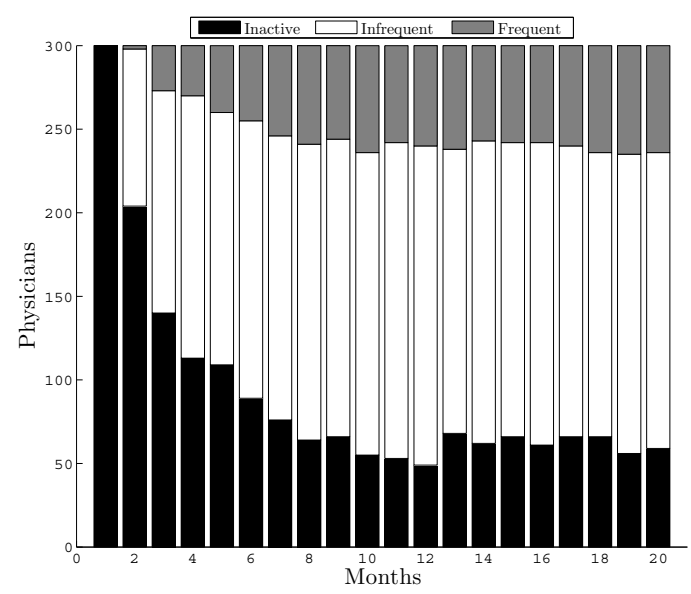

physicians' state membership to stabilize to approximately $28 \%, 51 \%$, and $21 \%$ in the inactive, infrequent, and frequent states respectively.

(iii) Disentangling the Short- and Long-Term Effects of Marketing Activities. The proposed HMM allows us to disentangle the total effect of marketing activities into two components: immediate and enduring effects. The immediate impact of detailing and sampling can be assessed by the effect that these activities have on the share of prescriptions conditional on being in a particular state (see Equation (16) and Table 6). On the other hand, the enduring effect of detailing and sampling can be assessed by their effect on the states transition matrix (see Equations (2) and (3) and Table 7).

The results in Tables 5 and 6 show that detailing and sampling have a relatively small short-term effects. This result is consistent with the finding of Mizik and Jacobson (2004). Specifically, both sampling and detailing have their primary positive short-term impact on physicians in the inactive state. That is, consistent with prior research (e.g., Manchanda et al. 2005; Narayanan et al. 2005), we find that pharmaceutical marketing actions play a relatively large role in affecting adoption early on. In contrast, when physicians are in the frequent state, we find a small negative short-term effect for detailing on share of prescription. This result, though counterintuitive, is consistent with the finding of Manchanda and Chintagunta (2004), who suggest that physicians may be conscious about the pressure being put on them by the companies' sales force and the possible physicians' backlash due to excessive marketing exposure. ${ }^{15}$

\footnotetext{
${ }^{15}$ Another explanation which cannot be ruled out is that competitors may be aware of the favorable behavior of
} 
In contrast to the relatively small short-term effects of these marketing activities, detailing and sampling have significant enduring effects. The results in Table 5 and in the middle and right matrices in Table 7 show that detailing and sampling have substantial effects in switching physicians from lower states to higher ones. By comparing the transition matrix without marketing mix interventions (left side of Table 7), with detailing only (center of Table 7), and with sampling only (right side of Table 7), we can see that detailing has a strong effect in moving physicians away from the inactive state. Sampling, on the other hand, is less effective in moving physicians away from the inactive state, but is more effective in keeping them in the frequent state. Thus, while detailing may be more useful as an acquisition tool, sampling is more useful as a retention tool. A possible explanation for this result is that when physicians are in the inactive state, they are more receptive to new information about the drug. Then, when they move to the infrequent and frequent states, there is diminishing return to additional information about the new drug. In the frequent state, physicians (and their patients) can primarily benefit from receiving free samples to encourage them to keep on prescribing the new drug.

Detailing and sampling elasticities. To analyze the overall impact of detailing and sampling, we compute the elasticities of new prescriptions with respect to each marketing activity. Average elasticities are computed numerically using the formula: $e_{y, x}=\frac{\Delta y / y}{\Delta x / x}$, where $y$ corresponds to the average monthly number of new prescriptions and $x$ corresponds to the average monthly number of details or samples.

To compute the increase in prescriptions $(\Delta y)$, we use the parameter estimates from the HMM to simulate the effect of targeting one detail or one sample to each physician in the first month of the simulation (thus, $\Delta x=300=1$ detail (or sample) x 300 physicians). We first compute the base prescription of the new drug when no details or samples are allocated to the physicians $\left(y_{0}\right)$. Then, we compute the number of prescriptions during the next 20 months following the targeting of one detail or one sample $\left(y_{1}\right)$. Thus, $\Delta y=y_{1}-y_{0}$. To compute the short-term elasticities, we consider the effect of detailing or sampling in the first month only; whereas to compute the long-term elasticities, we consider the effect in the remaining 19 months.

Consistent with Tables 6 and 7 , the results in Table 8 show that the magnitude of

physicians in the frequent state, and consequently can increase their marketing efforts to those physicians. However, there is no evidence of pharmaceutical companies reacting in such a way. 
Table 8: Detailing and sampling elasticities

\begin{tabular}{lrrr}
\hline Marketing action & Short-term & Long-term & Total \\
\hline Detailing & 0.002 & 0.652 & 0.654 \\
Sampling & 0.021 & 0.232 & 0.253 \\
\hline
\end{tabular}

the short-term elasticities is negligible compared to the magnitude of the long-term elasticities for detailing and sampling. Furthermore, in the short-term, sampling has a stronger effect relative to detailing; whereas in the long-term, detailing has a stronger effect. The elasticities presented in Table 8 are consistent in magnitude with the elasticities reported by Manchanda and Chintagunta (2004), Mizik and Jacobson (2004), and Sismeiro et al. (2007). Our results suggest that studies that consider only the short-term effect of detailing and sampling, significatively underestimate the impact of these marketing activities.

Magnitude and duration of the marketing actions effects. To assess the magnitude and duration of the impact of detailing and sampling, we simulate their effects using the same procedure we use for elasticity calculation. Relative to a no detailing and no sampling policy, we find that one detail (sample) produces on average 0.135 (0.0337) additional prescriptions during the first two months. In the long run, one detail (sample) produces 0.55 (0.11) additional prescriptions. Figure 4 shows the percentage increase in prescriptions over time.

Figure 4: Duration of the effect of marketing actions

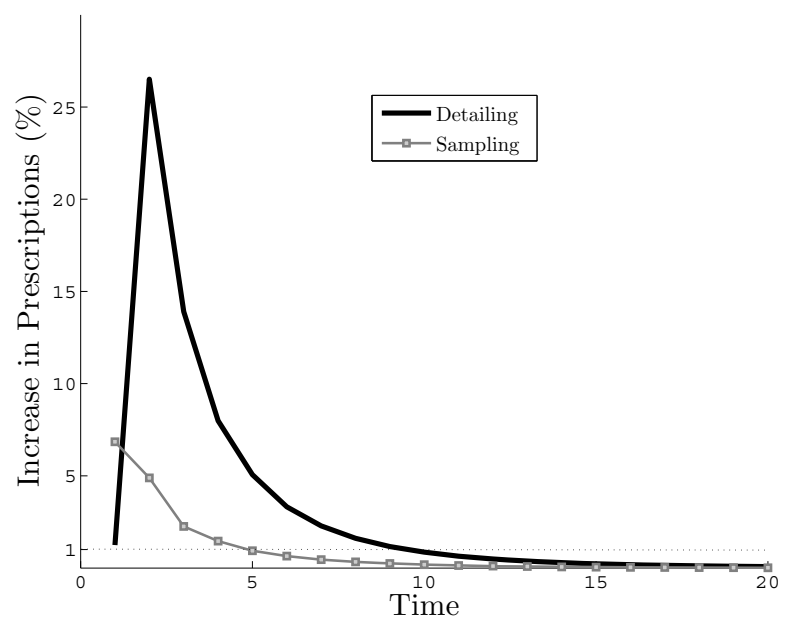


We observe that in the long-run the effect of detailing is both stronger in magnitude and longer in duration relative to the effect of sampling. In particular, the effect of detailing in the long-term is about 5 times the effect of sampling. However, the short-term (one month) effect of sampling is 5.5 times that of detailing. The duration of the effectiveness of detailing and sampling is 10 and 5 months, respectively; after which, the increase in prescription due to the marketing action is less than $1 \%$. Moreover, only 25\% (35\%) of the total effect of detailing (sampling) occurs in the first two months.

(iv) Heterogeneity Across Physicians. Thus far, we have presented the results at the aggregate level. However, the MCMC procedures and the hierarchical Bayesian estimation allow us to analyze the dynamics and effectiveness of the marketing activities at the physician level. For each parameter estimate we have the complete distribution across physicians. These parameters show a high degree of heterogeneity. Figure 5 depicts the heterogeneity in the transition matrix parameters across physicians (computed at the mean of detailing and sampling).

Figure 5: Heterogeneity in the transition matrix parameters across physicians
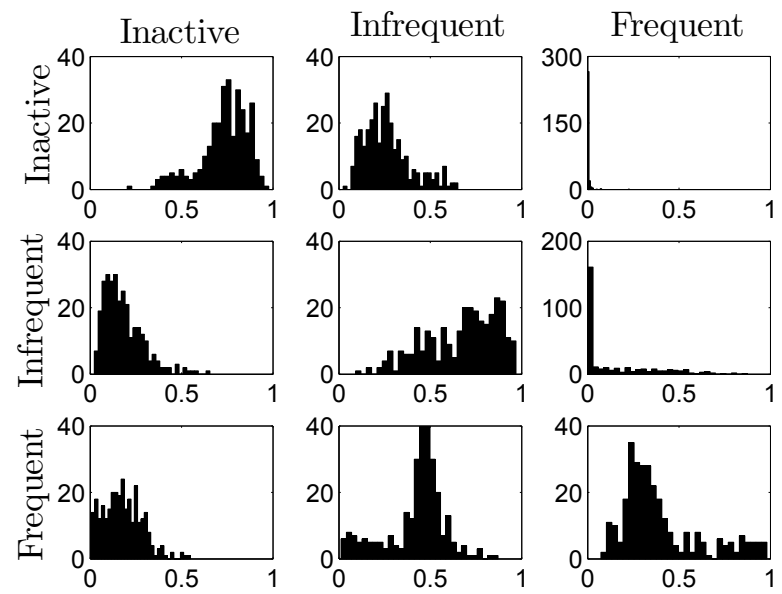

It is interesting to note that despite the average relative stickiness of the inactive and infrequent states, there is a group of physicians who are less sticky to those states. Similarly, the probability distribution of staying in the frequent state has a heavy right tail, suggesting that there is a group of physicians that is sticky to this state. 
In the next section, we use these individual-level estimates and present the third component of our integrative framework: the optimal marketing resource allocation across physicians and over time.

4.6.4. Optimal Marketing Mix Allocation. After capturing the physicians' heterogeneous and dynamic prescription behavior and having assessed the short- and long-term effects of detailing and sampling, the firm can optimally schedule future interventions at the physician level. Given that it is very costly to target all physicians, the pharmaceutical company needs to decide which physicians to contact, when to contact them, and how many details or samples they will receive each month for a given planning horizon. In this section we present the answer to these questions using two common managerial resource allocation scenarios: (i) given a fixed budget, how should the firm target and distribute its marketing resources across physicians? (Constrained dynamic programming problem); and (ii) given the firm's cost structure, what should the optimal budget and marketing mix allocation be? (Unconstrained dynamic programming problem). For each method, we describe the motivations, the proposed targeting policy, and the implications on the short- and long-term profits.

We assume that the pharmaceutical company needs to schedule its marketing interventions for a planning horizon of four months, which corresponds to the last four months in the data that we left out for holdout prediction purposes. ${ }^{16}$ The firm needs to determine the number of details and product samples each physician should receive each month in the planning horizon.

Optimal resource allocation with budget constraints. In many marketing resource allocation problems, the budget that should be allocated across customers is predetermined due to resource availability, budgetary constraints, or historical reasons. In such cases, the optimization task involves deciding how to allocate these resources given the budget constraints. This task corresponds to the problem described in Section 3.3. We determine the optimal dynamic policy, compute its performance and compare it to the performances obtained by applying either the current policy observed in the data; or a static policy, which only considers the short-term effects of marketing interventions and neglects physicians' dynamics in the planning horizon. To compute the implication of each policy, we simulate the effect of applying each policy on physicians' behavior for the planning horizon of four months. Additionally, to establish some base-level comparison, we analyze the implications of applying a no-marketing-interventions

\footnotetext{
${ }^{16}$ The method could be easily extended to longer planning horizons.
} 
policy, where we assume that the firm will not provide any detail or sample to any physician during the planning horizon. In solving the DP problem, we make the following assumptions: ${ }^{17}$ retail price of a prescription (including refills) $p=\$ 300$; cost of one detail $c_{d}=\$ 50$; cost of one sample $c_{s}=\$ 20 ; \delta=0.9$, which corresponds to a discount rate of $10 \%$. The profit $r_{i s t}$ in Equation (12) can be specified as: $r_{i s t}=p W_{i t} p_{i s t}-c_{d}$ Detailing $_{i t}-c_{s}$ Sampling $_{i t}$, where $W_{i t}$ is the total number of prescriptions in the category written by physician $i$ in month $t$; and $p_{i s t}$ is the share-of-prescriptions of the new drug allocated by physician $i$ at month $t$ (see Equations (15) and (16)).

The static policy is obtained by solving a multidimensional Knapsack problem for all physicians simultaneously each period independently, where physicians receive details and samples depending upon their responsiveness to these activities until the budget is deployed. The forward-looking policy is obtained by solving such a multidimensional Knapsack problem over 10 months, while considering the implications of firm decisions on future periods using a DP problem. In the DP problem, the periods are linked by physicians' dynamic responsiveness to the allocated resources. The multidimensional Knapsack problem is known to be NP-Hard and it has been previously studied in different fields. We apply a greedy heuristic to find a starting point from which we search for an optimal solution (see Appendix A for further details).

To illustrate the constrained optimization problem, we use the actual total number of details and samples offered to physicians each month during the last four months of the data as a budget constraint. Therefore, the task for each policy is to use that fixed budget and determine who receives details and samples, how many, and when. ${ }^{18}$ Table 9 shows the performances of applying such policies.

The results in Table 9 show that the current budget can be better allocated by implementing a policy which incorporates physicians' dynamics and the long-term effect of marketing actions. A dynamic forward-looking policy increases the number of prescriptions by $50.7 \%$ (2,947 vs. $1,955)$ relative to the current policy; and by $5.1 \%(2,947$ vs. 2,803$)$ relative to a static-myopic

\footnotetext{
${ }^{17}$ These estimates were determined based on discussions with the data provider and based on prior literature. The cost of one detail considers that three drugs are discussed during a 10-15 minutes visit. Sampling costs include the drug itself, packaging, shipping, and storing costs. Based on treatment specifications for this condition, we assume one new prescription corresponds to a treatment of three months on average. That is, an average patient needs to obtain two additional refills of the drug. The procedure presented in this section could be easily repeated given an alternative cost structure.

${ }^{18} \mathrm{We}$ assume that the feasible ranges for the number of details and samples are equal to the $95 \%$ percentile observed in the data. Additionally, we impose that each physician needs to be detailed in order to receive a sample. This constraint was observed in our data.
} 
Table 9: Constrained resource allocation policies results

\begin{tabular}{|c|c|c|c|}
\hline Policy & Prescriptions & $\begin{array}{l}\text { Prescription } \\
\text { increase }\end{array}$ & Profits \\
\hline Current & 1,955 & $10.1 \%$ & $\$ 380,349$ \\
\hline Static & 2,803 & $57.9 \%$ & $\$ 634,617$ \\
\hline Dynamic & 2,947 & $66.0 \%$ & $\$ 677,880$ \\
\hline
\end{tabular}

${ }^{*}$ The percentage increase in the number of prescriptions for each policy is relative to a no details and samples policy. The overall number of details and samples allocated to the 300 physicians during the 4 -month period is 2,244 and 4,701 , respectively.

policy. In other words, by considering the dynamic and long-term effect of detailing and sampling the firm can increase the total profits by $78 \%$ ( $\$ 677,880$ vs. $\$ 380,349)$ and the profits from each physician by $\$ 248=(\$ 677,880-\$ 380,349) /(300 x 4)$ per month with no additional marketing investment (the marketing budget is identical across policies). Considering that pharmaceutical companies typically target as many as 100,000 physicians, these additional earnings may amount to $\$ 24.8$ million per month. We will discuss further the insights on how these policies differ later in the section.

Optimal resource allocation without budget constraints. In the previous sub-section we assumed fixed budget constraints. However, our optimization framework can be used to determine the optimal budget as well. To do so, we solve the unconstrained DP problem described in Section 3.3 and compare its performance to the performance obtained by applying either the current or the static policies discussed previously.

Table 10: Unconstrained resource allocation policies results

\begin{tabular}{|c|c|c|c|c|c|c|}
\hline Policy & Prescriptions & $\begin{array}{l}\text { Prescription } \\
\text { increase }\end{array}$ & Details & Samples & Budget & Profits \\
\hline Current & 1,955 & $10.1 \%$ & 2,244 & 4,701 & $\$ 206,220$ & $\$ 380,349$ \\
\hline Static & 2,587 & $45.7 \%$ & 1,340 & 2,540 & $\$ 117,800$ & $\$ 658,231$ \\
\hline Dynamic & 2,797 & $57.5 \%$ & 1,563 & 3,766 & $\$ 153,470$ & $\$ 685,696$ \\
\hline
\end{tabular}

Several interesting implications can be drawn from Table 10. First, our results suggest that the pharmaceutical firm is currently overspending on detailing and sampling. Under the proposed dynamic policy, the firm should cut its detailing allocation by $30 \%(1,563$ 
vs. 2,244), its sampling allocation by $20 \%$ (3,766 vs. 4,701), and its overall spending by $26 \%$ ( $\$ 153,470$ vs. $\$ 206,220)$. These results are directionally consistent with the finding of Mizik and Jacobson (2004). Second, despite the 26\% cut in spending on marketing activities, the firm can generate $47.4 \%$ (57.5\% vs. $10.1 \%$ ) more prescriptions using the dynamic policy instead of the current policy, generating an additional $\$ 305,347$ in profits from our sample of 300 physicians over a period of four months. The increase in prescription and the decrease in marketing spending lead to an increase of $80 \%$ (685,696 vs. $380,349)$ in profitability relative to the current policy. The optimal budget, then, is about $\$ 128=\$ 153,470 /(300 \mathrm{x} 4)$ per physician per month ( $\$ 91$ on details and $\$ 37$ on samples). Third, a static policy underestimates the overall effect of detailing and sampling as it only incorporates their short-term effect. As a result, it allocates much less details and samples to physicians compared to the other policies.

The results of these resource allocation analyzes highlight the possibly substantial financial implications of simultaneously capturing the dynamics in consumer behavior and the long-term effect of marketing actions in marketing mix allocation.

Targeting, resource allocation, and timing implications. The optimization analysis described above suggests that using the dynamic policy, which takes advantage of the dynamics in prescription behavior and the enduring effect of marketing activities, the firm can significatively increase the return on marketing investments. To further understand the drivers of the improved performance of the proposed policy, we investigate the differences among the policies in terms of: who is being targeted, how the resources are allocated, and when these resources are distributed across physicians. As the results in Table 11 suggest, the current, dynamic constrained, and dynamic unconstrained policies, allocate similar number of details and samples to targeted physicians. However, the current policy targets a higher percentage of physicians (85\%) than the dynamic constrained (60\%) and dynamic unconstrained (50\%) policies. Furthermore, the overlap in the physicians targeted by the two dynamic policies is very high (over 90\%).

Given the congruency between the two dynamic policies, we compare only the current policy to the dynamic unconstrained policy. We compare these policies in terms of: (i) targeted physicians, (ii) allocated resources, and (iii) timing of targeting. 
Table 11: Policy comparisons

\begin{tabular}{lccc}
\hline Policy & Details $^{*}$ & Samples $^{*}$ & $\begin{array}{c}\text { Percentage of } \\
\text { physicians targeted }\end{array}$ \\
\hline Current & 2.21 & 10.26 & $85 \%$ \\
Dynamic constrained & 3.09 & 11.78 & $60 \%$ \\
Dynamic unconstrained & 2.61 & 9.78 & $50 \%$ \\
\hline
\end{tabular}

${ }^{*}$ Values correspond to the mean number of details and samples allocated to targeted physicians.

(i) Who is being targeted. The current policy presents limited discrimination among physicians. It targets about $85 \%$ of the physicians relative to only $50 \%$ of the dynamic (unconstrained) policy. In Table 12, we compare the physicians targeted by the dynamic policy with the physicians targeted by the current policy in terms of their short- and long-term responsiveness (elasticities) to marketing actions. We compute the average elasticities across the physicians targeted by each policy by simulating the effect of spending one detail or one sample in the first period of the planning horizon, as we did in Section 4.6.3 (see Appendix C). Additionally, we provide the elasticities for a no targeting policy which does not discriminate among physicians and offers details and samples to all physicians.

Table 12 shows that the dynamic policy targets physicians with substantially higher response to detailing and sampling than the current policy does. Furthermore, the elasticities corresponding to the current targeting do not differ substantially from those corresponding to a no targeting policy, supporting our finding of limited endogeneity in the current policy.

Table 12: Average elasticities across policies

\begin{tabular}{lccccccccc}
\hline \multirow{2}{*}{$\begin{array}{l}\text { Marketing } \\
\text { action }\end{array}$} & \multicolumn{3}{c}{ No targeting } & \multicolumn{3}{c}{ Current } & \multicolumn{3}{c}{ Dynamic } \\
\cline { 2 - 9 } ST $^{a}$ & LT $^{b}$ & Total $^{c}$ & ST & LT & Total & ST & LT & Total \\
\hline Detailing & -0.122 & 0.129 & 0.006 & -0.113 & 0.122 & 0.008 & 0.105 & 0.168 & 0.273 \\
Sampling & 0.080 & 0.218 & 0.298 & 0.069 & 0.223 & 0.292 & 0.157 & 0.254 & 0.411 \\
\hline
\end{tabular}

${ }^{a}$ ST denotes the short-term elasticity computed for the first period in the planning horizon.

${ }^{b}$ LT denotes the long-term elasticity computed for the remaining three periods in the planning horizon.

${ }^{c}$ Total denotes the elasticity computed for the four periods in the planning horizon.

(ii) How to allocate resources. Next, we investigate how resources are allocated for 
physicians in the three HMM states. Figure 6 illustrates the allocation of detailing and sampling across physicians in the three HMM states for the planning horizon of four months, by the current and the dynamic (unconstrained) policy.

Figure 6: Resources allocated across physicians in the three HMM states

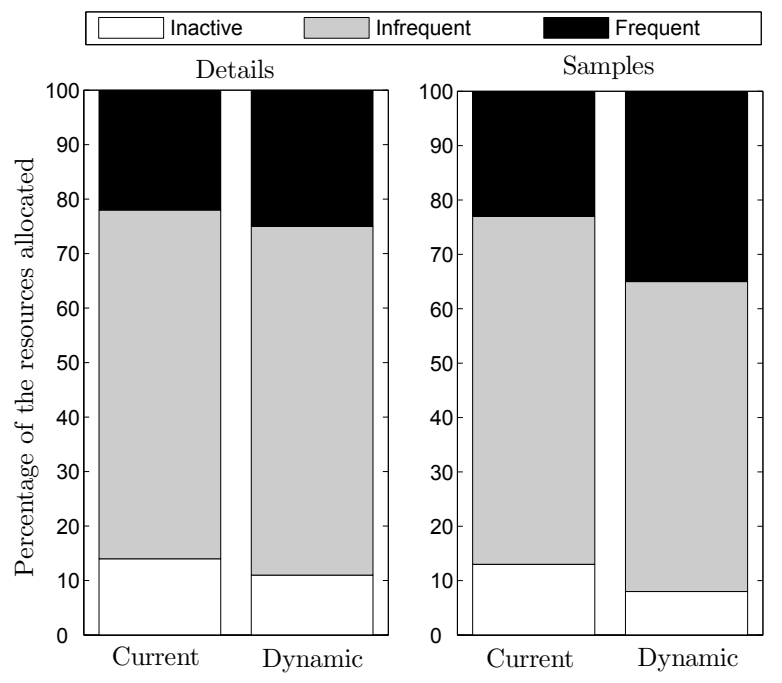

The main difference between the two policies is in the allocation of sampling. The dynamic policy, which allocates less resources overall (see Table 10), is particularly intensive in allocating samples to physicians in the frequent state (35\% of the total versus $23 \%$ by the current policy). This policy also allocates less resources to physicians in the inactive state. Indeed, Table 7 suggests that physicians in the frequent state are generally more responsive to sampling than to detailing. It is interesting to note that the dynamic policy allocates a significant amount of details to physicians in the frequent state even though, on average, physicians are not responsive to detailing in the frequent state. This may be due to the industry constraint that requires sales representatives to contact physicians through detailing in order to give them free samples, making the detail profitable due to the effectiveness of sampling during the detail visit.

(iii) When to distribute resources. Because of the dynamic structure of our model and the ability to capture the enduring impact of marketing actions, the proposed dynamic policy is capable of optimizing the timing of the marketing activities allocation to each physician. Figure 7 shows the percentage of physicians, in each of the 
HMM states, that are targeted each month in the planning horizon by each policy. This can be understood as the probability that a physician is targeted if she is in a particular state. We can observe in (a) that the current policy targets approximately $85 \%$ of the physicians every month regardless of the physicians' prescription states. Furthermore, the current policy is relatively static in the sense that similar policy is employed throughout the four months of the planning horizon.

Figure 7: Physicians targeted over time, across policies, and HMM states

\begin{tabular}{|l|l|l|}
\hline$\square$ Inactive $\square$ Infrequent $\square$ & Frequent \\
\hline
\end{tabular}

(a) Current

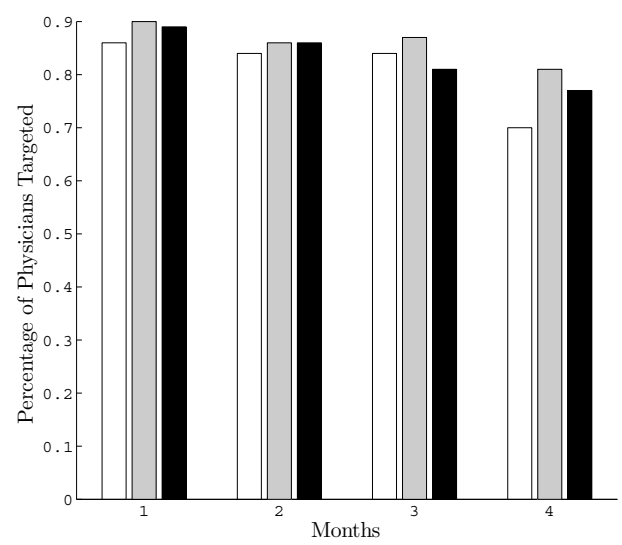

(b) Dynamic

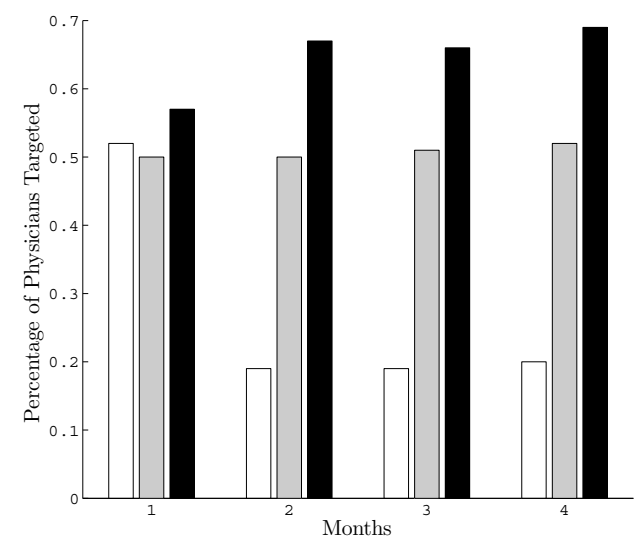

In contrast, the dynamic policy in (b) focuses on fewer physicians (50\%). Additionally, this policy exhibits interesting dynamics. In the first month, the dynamic policy targets physicians equally in the three HMM states. However, over time the dynamic policy focuses its targeting on physicians in the frequent state, and decreases its targeting to physicians in the inactive state. Thus, the dynamic policy seems to push physicians up the states. It first moves physicians from the inactive and infrequent states to the frequent state and then tries to keep these physicians in the frequent state.

In summary, the substantial increase in profits of the proposed dynamic policy relative to the current policy, can be attributed to the targeting of physicians with higher responsiveness to marketing actions, to the allocation of marketing actions differently 
across physicians based on the physician state of prescription, and to the use of detailing as an acquisition tool and sampling as a retention tool.

\section{Conclusions}

Managing customers through marketing mix allocation has a critical impact on firms' profitability in the long-term. To successfully target marketing interventions at the individual level, firms need to consider customers' evolution over time, heterogeneity in customers' dynamics and response to marketing mix interventions, and the possibly enduring effect of these marketing activities.

In this paper, we present an integrative framework to capture the dynamics in customer behavior and long-term effect of marketing activities at the individual level. We subsequently propose a dynamic programming approach to optimize the marketing mix allocation for long-term profitability. We identify several interesting findings by applying our modeling framework in the context of a pharmaceutical new drug introduction. First, we identify three latent prescription-behavior states which characterize physicians' dynamic behavior. Second for the particular drug studied, both detailing and sampling have long-term impact on physicians' prescription behavior. Third, detailing is particularly effective as an acquisition tool, moving physicians from an inactive state, whereas sampling is mostly effective as a retention tool, keeping physicians in a high prescriptionbehavior state. Fourth, sampling has a stronger short-term effect than detailing, but the latter has a stronger long-term effect. Fifth, we demonstrate that ignoring the dynamics in physician buying behavior and the long-term effects of marketing activities leads to suboptimal allocation of marketing interventions. Specifically, using a counterfactual analysis, we demonstrate that our resource allocation approach leads to an increase of $80 \%$ in profitability and that the firm should cut its marketing spending by $26 \%$. Finally, the optimal dynamic allocation of sampling and detailing involves first moving physicians away from the inactive state to the frequent state and then retaining these physicians in the frequent state.

We highlight several limitations and directions that future research could explore. 
First, in this empirical application, we find no evidence of endogeneity in the detailing and sampling for the specific drug. In general, if endogeneity is present, one could integrate into our modeling approach a targeting process equation along the lines of Manchanda et al. (2004). Second, an alternative source of dynamics not considered in this research comes from the belief that physicians have foresight regarding their prescription-behavior evolution and the firm's marketing mix allocation. One could extend our modeling framework by formulating a structural model of state-dependence with forward-looking behavior (Erdem and Keane 1996). Given the nature of dynamics in HMMs, this is likely to complicate the modeling approach significatively. Third, similar to many CRM data sets, our data include no information about competitive marketing efforts. Obtaining such information would be helpful to describe physicians' dynamics. Alternatively, one could use the HMM approach of Moon et al. (2007) to augment the competitive marketing efforts. Fourth, the proposed framework can be applied to many CRM applications such as loyalty programs, contractual services, and new product introduction. In these type of applications, firms need to trade-off between short- and long-term revenues when allocating marketing resources at the individual level.

To summarize, firms are engaged in developing marketing initiatives that are aimed at enhancing the relationship between the customer and the firm, and thus have an enduring impact on the customer. Optimal targeting and allocation of such marketing activities is extremely difficult. In this research, we offer a first step in providing marketing managers a framework for managing customers for long-run profitability through an efficient dynamic allocation of marketing resources. 


\section{Appendix A. Algorithm for Solving the Dynamic Programming}

\section{Problem}

In this Appendix we describe the details of how we solve the Dynamic Programming (DP) problems of dynamic marketing mix allocation. Following Section 3.3, the firm's dynamic marketing allocation problem can be formulated as:

$$
\begin{aligned}
\sum_{i=1}^{N} V_{i}^{*}\left(\mathbf{B}_{i t}\right) & =\sum_{i=1}^{N} \max _{\mathbf{z}_{i t}} E\left\{\sum_{\tau=t}^{\infty} \delta^{\tau-t} R_{i \tau}\right\} \\
& =\sum_{i=1}^{N} \max _{\mathbf{z}_{i t}}\left\{\sum_{s=1}^{S} b_{i t}(s) \cdot r_{i s t}+\delta\left[V_{i}^{*}\left(\mathbf{B}_{i t+1}\right)\right]\right\},
\end{aligned}
$$

subject to

$$
\begin{aligned}
\mathbf{B}_{i t+1} & =\mathbf{B}_{i t} \mathbf{Q}_{i t} & \forall i, t & & \text { (update beliefs) } \\
\sum_{i=1}^{N} \mathbf{z}_{i t} & \leq \mathbf{A}_{\mathbf{t}} & \forall t & & \text { (marketing budget) } \\
\mathbf{z}_{i t} & \in \mathbf{D} & \forall i, t & & \text { (marketing actions space) }
\end{aligned}
$$

it is important to note that the state variables in our DP problem $\left(\mathbf{B}_{i t}\right.$, beliefs about customer $i$ at time $t$ ) are continuous. Therefore, in contrast to problems with a finite number of state points, it is not possible to solve exactly for $V_{i}^{*}\left(\mathbf{B}_{i t}\right)$ at every state point. Thus, we employ an approximation method. Specifically, we follow Keane and Wolpin (1994) by using a polynomial approximation for $V_{i}^{*}\left(\mathbf{B}_{i t}\right)$ as we describe below.

Given that the firm's beliefs about customers' membership to the HMM states add up to one, the second order polynomial ${ }^{19}$ for $V_{i}^{*}\left(\mathbf{B}_{i t}\right)$ for the three-state HMM problem used in our empirical application is a function of the beliefs about customer $i$ 's membership to state 1 and 2 only. Specifically:

$$
V_{i}^{*}\left(\mathbf{B}_{i t}\right)=\gamma_{i 0}+\gamma_{i 1} b_{i t}(1)+\gamma_{i 2} b_{i t}^{2}(1)+\gamma_{i 3} b_{i t}(2)+\gamma_{i 4} b_{i t}^{2}(2)+\gamma_{i 5} b_{i t}(1) b_{i t}(2),
$$

where $b_{i t}(s)$ corresponds to the firm's belief about customer $i$ 's membership to state $s$ at time $t$. The parameters $\left\{\gamma_{i j}\right\}_{j=0}^{5}$ are estimated by OLS regression for each customer $i$. To obtain the sample of data points (belief points) for the interpolation, we calculate $V_{i}^{*}\left(\mathbf{B}_{g}\right)$ on a grid of $N_{G}=100$ belief points different for each customer $i$ but constant over time. ${ }^{20}$ To reduce the complexity of the optimization algorithm, we set up the grid by selecting a sample of beliefs only from a "relevant" space of beliefs for the customer. The relevant space is defined by the firm's beliefs about customer $i$ 's membership at time $t$ and the possible transitions for that customer given the range of marketing actions in each period. For instance, if the firm's beliefs about customer $i$ 's membership are $\mathbf{B}_{i t}=[0.8,0.2,0]$. Then, by allocating the minimum number of marketing actions to this customer at period $t$, its beliefs could be updated for the next period to be $\mathbf{B}_{i t+1}^{\min }=[1,0,0]$. On the other extreme, by allocating the maximum number of marketing actions to this customer at period $t$, its beliefs could be updated for the next period to be

\footnotetext{
${ }^{19}$ One could define a higher order polynomial. However, for our empirical application a higher order polynomial did not provide significantly better interpolation results.

${ }^{20}$ Larger $N_{G}\left(N_{G}=200,500\right.$, and 1,000$)$ did not provide significant improvement in fit or predictability.
} 
$\mathbf{B}_{i t+1}^{\max }=[0.5,0.3,0.2]$, then only beliefs $\mathbf{B}_{i t+1} \in\left[\mathbf{B}_{i t+1}^{\min }, \mathbf{B}_{i t+1}^{\max }\right]$ will be feasible. We randomly draw $N_{G}$ i.i.d. of those feasible beliefs.

Having defined the grid over which we calculate the value functions, we next describe the backsolving method used to solve the DP problems. For ease of exposition, we first describe the algorithm used for solving the resource allocation with budget constraints problem, and then, the algorithm used for solving the resource allocation without budget constraints problem.

Optimal Resource Allocation With Budget Constraints. For the following algorithm we assume a finite horizon $T$, and a size $N_{G}$ for the random grid of beliefs. Each period $t$ we create a random grid for customer $i, G_{i t}=\left\{\xi_{i, t, j}\right\}_{j=1}^{N_{G}}$, which represents the firm's beliefs about customer $i$ 's state membership at time $t$. In order to solve the DP problem we employ a procedure which consists of backsolving the DP problem starting from the last period imposing the known budget constraints for that period. We find the optimal resource allocation across customers for that period (solving a knapsack problem) and, then, use the estimated value functions $V_{i}^{*}\left(\mathbf{B}_{i T}\right)$ to run the OLS regression and estimate the $\gamma_{i j}$ coefficients in Equation (A.2) which are used to approximate the value function in the next step. Then, for period $T-1$, we use the interpolated $V_{i}^{*}\left(\mathbf{B}_{i T}\right)$ in order to optimally solve for $V_{i}^{*}\left(\mathbf{B}_{i T-1}\right)$. We repeat this procedure up to time 1. At the initial period of the planning horizon, $\mathbf{B}_{i 1}$ correspond to the firm's beliefs of customer $i$ 's state membership at the end of the calibration period. Thus, we use these probabilistically "known" beliefs to forward allocate the marketing resources following the optimal path determined by the backsolving procedure of the DP algorithm. To further clarify the employed procedure, we present a description of the algorithm used: ${ }^{21}$

1. (Backsolving) Last period $T$

(a) Create the grid of state-space (beliefs) points $G_{i T}=\left\{\xi_{i, T, j}\right\}_{j=1}^{N_{G}} \forall i$. The entire grid across customers $\mathbf{G}_{T}$ is of size $N_{G}$ x $N$, where $\mathrm{N}$ is the number of customers.

(b) For each set of beliefs $g \in \mathbf{G}_{T}$ solve a knapsack problem to allocate marketing resources across customers.

(c) Compute the value function $V_{i T g}^{*}$ for each customer $i$ given the optimal solution reached for grid point $g$.

(d) Use $\left\{V_{i T g}^{*}\right\}_{g=1}^{N_{G}}$ as dependent variable in the OLS regression to obtain the $\gamma_{i j}$ parameters, and approximate $\widehat{V}_{i T}^{*}$ for any state beliefs.

2. Period $t=T-1, \ldots, 2$

(a) Create the grid of state-space (beliefs) points $G_{i t}=\left\{\xi_{i, t, j}\right\}_{j=1}^{N_{G}} \quad \forall i$.

(b) For each set of beliefs $g \in \mathbf{G}_{t}$ solve a knapsack problem to allocate marketing resources across physicians considering short- and long-term effects. Use the approximation $\widehat{V}_{i t+1}^{*}$, in the Bellman equation, to compute $V_{i t}^{*}$.

(c) Compute the value function $V_{i t g}^{*}$ for each customer $i$ given the optimal solution reached for grid point $g$.

\footnotetext{
${ }^{21}$ For illustration purposes and to simplify notation, we write $V_{i}^{*}\left(\mathbf{B}_{i t}\right)$ as $V_{i t}^{*}$ in the following description.
} 
(d) Run the OLS regression to obtain the $\gamma_{i j}$ parameters and approximate $\widehat{V}_{i t-1}^{*}$ for any state beliefs.

(e) Make $t=t-1$ and repeat (a)-(d) until $t-1=1$.

3. Period 1 (forward allocation). Allocate the marketing resources using $\mathbf{B}_{i 1}$, the optimal policy found for these beliefs, and the approximation $\widehat{V}_{i 2}^{*}$ obtained in period 2 using:

$$
V_{i 1}\left(\mathbf{B}_{i 1}\right)=\sum_{s=1}^{S} b_{i 1}(s) r_{i s t}+\delta \widehat{V}_{i 2}^{*}\left(\mathbf{B}_{i 2}\right)
$$

and solving the Knapsack problem in Equation (A.1).

After determining the optimal policy for period 1, update beliefs and move to period 2. Repeat until terminal period $\mathrm{T}$, where by assumption $\widehat{V}_{i T+1}^{*} \equiv 0$ for all beliefs and customers.

Optimal Resource Allocation Without Budget Constraints. This case is solved similarly to the constrained case except for two modifications: (i) there is no common budget constrains across customers. Therefore, the problem is solved independently for each customer; (ii) we solve an infinite DP problem. We do so, by implementing a value iteration policy, which consists on backsolving the DP problem for a sufficiently large number of periods and until no major change is observed in the value function for each individual. Specifically, we backsolve the DP problem until we reach a point where, in going back one period, the maximum percentage change in the value function across all grid points - which is measured by the change in the polynomial parameters $\gamma_{i j}$ - is less than $0.1 \%$. In our empirical application, the mean number of time periods (iterations of the value iteration method) required until convergence was 61.9 and the mean $R^{2}$ of the OLS regression for the interpolation method was 0.99 . We generate the random grid only once for each customer, and keep it until convergence.

Note that this procedure is impractical when there are budget constrains as the ones described previously, because a DP problem with budget constraints requires solving a knapsack problem across customers. This would have to be performed for each iteration of the value iteration method, which usually requires several iterations until convergence.

Dealing With Local Optima. The objective function of the DP problem described above is highly nonlinear in the decision variables. Therefore, any optimization routine is susceptible to find local optima. To reduce this concern, a few improvements were implemented. For the problem without budget constrains, we implemented an enumeration algorithm which computes and compares all feasible solutions and returns the global maximum. Given that this problem is solved individually, this routine is implementable in reasonable time. For the problem with budget constrains, an enumeration algorithm is infeasible as the number of feasible solutions is prohibitive (any feasible solution can be implemented in $N$ ! ways, where $N$ is the number of customers). We therefore implemented a greedy heuristic to find a good starting point, from which the optimization routine starts searching for an optimal solution. This greedy heuristic consists on allocating resources iteratively based on the unit increase in the overall objective function. This allocation is made in decreasing order of improvement until the total budget is deployed. 


\section{Appendix B. Prior and Full Conditionals Distributions}

We denote by $\boldsymbol{\theta}_{i}=\left\{\tau_{i s 1}, \ldots, \tau_{i s S-1}, \boldsymbol{\rho}_{i s}, \boldsymbol{\alpha}_{i s}\right\}_{s=1}^{S}$ the set of random-effect parameters and by $\boldsymbol{\Phi}=\left\{\alpha_{s}^{0}\right\}_{s=1}^{S}$ the set of fixed-effect parameters. See Equations (3)-(16).

Priors

Random-effect parameters $\boldsymbol{\theta}_{i} \quad: \quad \boldsymbol{\theta}_{i} \backsim N\left(\boldsymbol{\mu}_{\theta}, \boldsymbol{\Sigma}_{\theta}\right) \Rightarrow P\left(\boldsymbol{\theta}_{i}\right) \propto \exp \left(\frac{1}{2}\left(\boldsymbol{\theta}_{i}-\boldsymbol{\mu}_{\theta}\right)^{\prime} \boldsymbol{\Sigma}_{\theta}^{-1}\left(\boldsymbol{\theta}_{i}-\boldsymbol{\mu}_{\theta}\right)\right)$
$\boldsymbol{\mu}_{\theta} \backsim N\left(\boldsymbol{\mu}_{0}, \mathbf{V}_{0}\right) \Rightarrow P\left(\boldsymbol{\mu}_{\theta}\right) \propto \exp \left(\frac{1}{2}\left(\boldsymbol{\mu}_{\theta}-\boldsymbol{\mu}_{0}\right)^{\prime} \mathbf{V}_{0}^{-1}\left(\boldsymbol{\mu}_{\theta}-\boldsymbol{\mu}_{0}\right)\right)$
$\boldsymbol{\Sigma}_{\theta} \backsim I W\left(d f_{0}, d f_{0} \mathbf{S}_{\theta}\right)$

Fixed-effect parameters $\boldsymbol{\Phi} \quad: \quad \boldsymbol{\Phi} \backsim N\left(\boldsymbol{\mu}_{\Phi}, \boldsymbol{\Sigma}_{\Phi}\right) \Rightarrow P(\boldsymbol{\Phi}) \propto \exp \left(\frac{1}{2}\left(\boldsymbol{\Phi}-\boldsymbol{\mu}_{\Phi}\right)^{\prime} \boldsymbol{\Sigma}_{\Phi}^{-1}\left(\boldsymbol{\Phi}-\boldsymbol{\mu}_{\Phi}\right)\right)$

Likelihood : $L\left(\right.$ data, $\left.\left\{\boldsymbol{\theta}_{i}\right\}, \boldsymbol{\Phi}, \boldsymbol{\mu}_{\theta}, \boldsymbol{\Sigma}_{\theta}\right)=P\left(\right.$ data $\left.\mid\left\{\boldsymbol{\theta}_{i}\right\}, \boldsymbol{\Phi}\right) P\left(\left\{\boldsymbol{\theta}_{i}\right\} \mid \boldsymbol{\mu}_{\theta}, \boldsymbol{\Sigma}_{\theta}\right) P(\boldsymbol{\Phi}) P\left(\boldsymbol{\mu}_{\theta}\right) P\left(\boldsymbol{\Sigma}_{\theta}\right)$

Full conditionals : $P\left(\boldsymbol{\theta}_{i} \mid \boldsymbol{\mu}_{\theta}, \boldsymbol{\Sigma}_{\theta}, \boldsymbol{\Phi}\right.$, data $\left._{i}\right) \propto \exp \left(\frac{1}{2}\left(\boldsymbol{\theta}_{i}-\boldsymbol{\mu}_{\theta}\right)^{\prime} \boldsymbol{\Sigma}_{\theta}^{-1}\left(\boldsymbol{\theta}_{i}-\boldsymbol{\mu}_{\theta}\right)\right) P\left(\operatorname{data}_{i} \mid \boldsymbol{\theta}_{i}, \boldsymbol{\Phi}\right)$

$P\left(\boldsymbol{\Phi} \mid \boldsymbol{\mu}_{\Phi}, \boldsymbol{\Sigma}_{\Phi},\left\{\boldsymbol{\theta}_{i}\right\}\right.$, data $) \propto \exp \left(\frac{1}{2}\left(\boldsymbol{\Phi}-\boldsymbol{\mu}_{\Phi}\right)^{\prime} \boldsymbol{\Sigma}_{\Phi}^{-1}\left(\boldsymbol{\Phi}-\boldsymbol{\mu}_{\Phi}\right)\right) P\left(\right.$ data $\left.\mid\left\{\boldsymbol{\theta}_{i}\right\}, \boldsymbol{\Phi}\right)$

$\boldsymbol{\mu}_{\theta} \backsim N\left(\boldsymbol{\mu}_{n}, \mathbf{V}_{n}\right)$

$\boldsymbol{\Sigma}_{\theta} \backsim I W\left(d f_{1}, d f_{1} \mathbf{S}_{1}\right)$

where,

$$
\begin{aligned}
\mathbf{V}_{n}^{-1} & =\left[\mathbf{V}_{0}^{-1}+N \boldsymbol{\Sigma}_{\theta}^{-1}\right] \\
\boldsymbol{\mu}_{n} & =\mathbf{V}_{n}\left[\boldsymbol{\mu}_{0} \mathbf{V}_{0}^{-1}+N \bar{\theta} \boldsymbol{\Sigma}_{\theta}^{-1}\right] \\
d f_{1} & =d f_{0}+N \\
d f_{1} \mathbf{S}_{1} & =\sum_{i=1}^{N}\left(\boldsymbol{\theta}_{i}-\boldsymbol{\mu}_{\theta}\right)\left(\boldsymbol{\theta}_{i}-\boldsymbol{\mu}_{\theta}\right)^{\prime}+d f_{0} \mathbf{S}_{0}
\end{aligned}
$$

$P\left(\right.$ data $\left.\mid\left\{\boldsymbol{\theta}_{i}\right\}, \boldsymbol{\Phi}\right)$ and $P\left(\operatorname{data}_{i} \mid \boldsymbol{\theta}_{i}, \boldsymbol{\Phi}\right)$ are given by Equation (7).

The MCMC procedure generates a sequence of draws from the posterior distribution of the model's parameters. Since the full conditionals for $\boldsymbol{\theta}_{i}$ and $\boldsymbol{\Phi}$ do not have a closed form, the Metropolis-Hastings (M-H) algorithm is used to draw the samples. In particular, we use a gaussian random-walk M-H where the proposal vector of parameters $\varphi^{(t)}$ for $\boldsymbol{\theta}_{i}$ or $\boldsymbol{\Phi}$ at iteration $t$ is drawn from $N\left(\varphi^{(t-1)}, \sigma^{2} \Delta\right)$ and accepted using the M-H acceptance ratio. The tuning parameters $\sigma$ and $\Delta$ are adaptively chosen to yield an acceptance rate of approximately 20\%. We use the method proposed by Atchadé(2006) to adapt these tuning parameters.

We use the following uninformative prior hyperparameters for the three-state HMM:

$\boldsymbol{\mu}_{0}=[-0.7,0.3,-0.7,0.3,-0.7,0.3, \xi], \mathbf{V}_{0}=(1 / 5) \mathbf{I}_{n \theta X n \theta}, d f_{0}=n \theta+5, \mathbf{S}_{0}=\mathbf{I}_{n \theta X n \theta}$,

$\boldsymbol{\mu}_{\Phi}=[0,-4,-4], \boldsymbol{\Sigma}_{\Phi}=\mathbf{I}_{n \Phi X n \Phi}$. Where $\xi$ is a $1 \times 12$ vector of zeros, $\mathrm{N}$ is the number of individuals, and $\mathbf{I}_{n a X n a}$ denotes an identity matrix of rank $n a$.

Note: An appropriate selection of the priors is particularly critical in this model since the parameters are transformed to an exponential scale. Moreover, we model heterogeneity using a hierarchical structure, where the variance of $\boldsymbol{\mu}_{\theta}$ and $\boldsymbol{\Sigma}_{\theta}$ are added at each individual draw of each $\boldsymbol{\theta}_{i}$. This increases the variance of the transformed variables. Accordingly, we chose prior hyperparameters for $\boldsymbol{\mu}_{\theta}, \boldsymbol{\Sigma}_{\theta}$, and $\boldsymbol{\mu}_{\Phi}$ such that their priors are diffused in the transformed space. 


\section{Appendix C. Simulation of the Model}

This Appendix describes how we simulate data from the model to compute the magnitude and duration of marketing effects in Section 4.6.3 and to evaluate the resource allocation policies described in Section 4.6.4.

Magnitude and Duration of the Marketing Effects. After estimating the parameters of the HMM we simulate the effects of sending a different number of details and samples to the physicians in the shortand long-term. We considered 20 months as our planning horizon. We simulate the effects of allocating no marketing resources in the entire planning horizon and compare it to sending either one detail or one sample to each customer in the sample in the first period only. That is, 300 details or 300 samples in period 1 and no details or samples in the next 19 periods. To compare these simulations, we assume physicians write their expected number of prescriptions given by the binomial model. Furthermore, we update the membership probabilities (beliefs $\mathbf{B}_{i t}$ ) in a deterministic manner using Equation (10) in Section 3.3. $\left(\mathbf{B}_{i t+1}=\mathbf{B}_{i t} \mathbf{Q}_{i t}\right)$. Because in our model detailing and sampling affect both the transition matrix $\mathbf{Q}_{i t}$ and the conditional choice matrix $\mathbf{M}_{i t}$, a different sequence of decisions are made based on differences in physicians' membership to the prescription-states and their propensity to prescribe the new drug conditional on being in a state.

Evaluating the Resource Allocation Policies. Having obtained the optimal resource allocation policies described in Section 4.6.4, we simulate the effects of implementing those policies in the sample of physicians. The implementation follows analogous steps to the ones described above, with two modifications: (i) we use a realistic four period planning horizon, which corresponds to the last four periods in the data (months 21-24), and (ii) the alternative policies imply different marketing resource allocation throughout the four periods (not only the first period of the planning horizon). 


\section{References}

Atchadé, Yves. 2006. An Adaptive Version for the Metropolis Adjusted Langevin Algorithm with a Truncated Drift. Methodology and Computing in Applied Probability, 8, 235-254.

Aviv, Yossi, Amit Pazgal. 2005. A Partially Observed Markov Decision Process for Dynamic Pricing. Management Science, 51(9), 1400-1416.

Bates, Andrée. 2006. Using ROI Data for Effective Decision Making in Pharmaceutical Marketing. KeywordPharma Expert Review.

Bawa, Kapil, Robert Shoemaker. 1987. The Effects of a Direct Mail Coupon on Brand Choice Behavior. Journal of Marketing Research, 24(4), 370-376.

Bellman, Richard. 1957. Dynamic Programming. Princeton University Press, Princeton.

Bitran, Gabriel, Susana Mondschein. 1996. Mailing Decisions in the Catalog Sales Industry. Management Science, 42(9),1364-1381.

Bucklin, Randolph, James Lattin. 1991. A Two-State Model of Purchase Incidence and Brand Choice. Marketing Science, 10(1), 24-39.

Chintagunta, Pradeep, Naufel Vilcassim. 1994. Marketing Investment Decisions in a Dynamic Duopoly: A Model and Empirical Analysis. International Journal of Research in Marketing, 11, 287-306.

Colombo, Richard, Weina Jiang. 1999. A Stochastic RFM Model. Journal of Interactive Marketing, 13(3), 2-12.

Dekimpe, Marnik, Dominique Hanssens. 1995. The Persistence of Marketing Effects on Sales. Marketing Science, 14(1), 1-21.

Dekimpe, Marnik, Dominique Hanssens. 1999. Sustained Spending and Persistent Response: A New Look at Long-Term Marketing Profitability. Journal of Marketing Research, 36(4), 397-412.

Dekimpe, Marnik, Dominique Hanssens, Vincent Nijs, Jan-Benedict Steenkamp. 2005. Measuring Short- and Long-Run Promotional Effectiveness on Scanner Data using Persistence Modeling. Applied Stochastic Models in Business and Industry, 21, 409416.

Djurić, Petar, Joon-Hwa Chun. 2002. An MCMC Sampling Approach to Estimation of Nonstationary Hidden Markov Models. IEEE Transaction on Signal Processing, 50(5), 1113-1123.

Du, Rex, Wagner Kamakura. 2006. Household Lifecycles and Lifestyles in America. Journal of Marketing Research, 43(1), 121-132.

Elsner, Ralf, Manfred Krafft, Arnd Huchzermeier. 2004. Optimizing Rhenania's direct marketing business through dynamic multilevel modeling (DMLM) in a multi-catalogbrand environment. Marketing Science, 23(2), 192-206.

Erdem, Tülim, Susumu Imai, Michael Keane. 2003. A Model of Consumer Brand and Quantity Choice Dynamics under Price Uncertainty. Quantitative Marketing and Eco- 
nomics, 1(1), 5-64.

Erdem, Tülim, Michael Keane. 1996. Decision-Making under Uncertainty: Capturing Dynamic Brand Choice Processes in Turbulent Consumer Goods Markets. Marketing Science, 15(1), 1-20.

Erdem, Tülim, Baohong Sun. 2001. Testing for Choice Dynamics in Panel Data. Journal of Business and Economic Statistics, 19(2), 142-152.

Gatignon, Hubert, Dominique Hanssens. 1987. Modeling Marketing Interaction with Application to Sales Force Effectiveness. Journal of Marketing Research, 24(3), 247-257.

Geisser, Seymour. 1993. Predictive Inference: An Introduction. Chapman and Hall, London.

Gelfand, A., D. Dey. 1994. Bayesian Model Choice: Asymptotics and Exact Calculations. Journal of the Royal Statistical Society, Series B, 56, 501-514.

Gelman, Andrew, Donald Rubin. 1992. Inference from Iterative Simulation Using Multiple Sequences. Statistical Science, 7, 457-511.

Gönül, Füsun, Franklin Carter, Elina Petrova, Kannan Srinivasan. 2001. Promotion of Prescription Drugs and Its Impact on Physicians Choice Behavior. Journal of Marketing, 65(3), 79-90.

Gönül, Füsun, Frenkel Ter Hofstede. 2006. How to Compute Optimal Catalog Mailing Decisions. Marketing Science, 25(1), 65-74.

Gönül, Füsun, Meng Ze Shi. 1998. Optimal Mailing of Catalogs: A New Methodology Using Estimable Structural Dynamic Programming Models. Management Science, 44(9), 1249-1262.

Greene, William. 1997. Econometric Analysis. Prentice Hall, Third edition.

Guadagni, Peter, John Little. 1983. A Logit Model Scanner Data. Marketing Science, $2(3), 203-238$.

Heckman, James. 1981. Heterogeneity and State Dependence. In S.Rosen ed. Studies in Labor Markets, Chicago: University of Chicago Press, 91-139.

Janakiraman, Ramkumar, Shantanu Dutta, Catarina Sismeiro, Phil Stern. 2005. Are They All the Same? Physicians' Persistence and Its Implications for Marketing Communication. Working paper, Tanaka Business School.

Jedidi, Kamel, Carl Mela, Sunil Gupta. 1999. Managing Advertising and Promotion for Long-Run Profitability. Marketing Science, 18(1), 1-22.

Kamakura, Wagner, Gary Russell. 1989. A Probabilistic Choice Model for Market Segmentation and Elasticity Structure. Journal of Marketing Research, 26(4), 379-390.

Keane, Michael. 1997. Modeling Heterogeneity and State Dependence in Consumer Choice Behavior. Journal of Business and Economic Statistics, 15(3), 310-327.

Keane, Michael, Kenneth Wolpin. 1994. The Solution and Estimation of Discrete Choice Dynamic Programming Models by Simulation and Interpolation: Monte Carlo Evidence. The Review of Economics and Statistics, MIT Press, vol. 76(4), 648-672. 
Kim, Chang-Jin, Charles Nelson. 1999. State-Space Models with Regime Switching: Classical and Gibbs Sampling Approaches with Applications. M.I.T. Press, Cambridge, Massachusetts.

Lewis, Michael. 2005. A Dynamic Programming Approach to Customer Relationship Pricing. Management Science, 51(6),986-994.

Liechty, John, Michel Wedel, Rik Pieters. 2003. The Representation of Local and Global Scanpaths in Eye Movements Through Bayesian Hidden Markov Models. Psychometrika, 68(4), 519-541.

Lovejoy, William. 1991. Computationally Feasible Bounds for Partially Observed Markov Decision Processes. Operations Research, 39(1), 162-175.

Manchanda, Puneet, Pradeep Chintagunta. 2004. Responsiveness of Physician Prescription Behavior to Salesforce Effort: An Individual Level Analysis. Marketing Letters, 15(2-3), 129-145.

Manchanda, Puneet, Peter Rossi, Pradeep Chintagunta. 2004. Response Modeling with Nonrandom Marketing-Mix Variables. Journal of Marketing Research 41(4), 467-478.

Manchanda, Puneet, Ying Xie, Nara Youn. 2005. The Role of Targeted Communication and Contagion in Product Adoption. Working paper, Graduate School of Business, University of Chicago.

Mantrala, Murali. 2002. Allocating Marketing Resources. Barton A. Weitz, Robin Wensley, eds. Handbook of Marketing. Sage Publications, Thousand Oaks, CA, 409-435.

McDonald, Iain, Walter Zucchini. 1997. Hidden Markov and Other Models for Discrete Valued Time Series. Chapman and Hall, London.

Mela, Carl, Sunil Gupta, Donald Lehmann. 1997. The Long-term Impact of Promotion and Advertising on Consumer Brand Choice. Journal of Marketing Research, 34(1), 248-261.

Mizik, Natalie, Robert Jacobson. 2004. Are Physicians 'Easy Marks'? Quantifying the Effects of Detailing and Sampling on New Prescriptions. Management Science, 50(12), $1704-1715$.

Monahan, George. 1982. A Survey of Partially Observable Markov Decision Processes: Theory, Models, and Algorithms. Management Science, 28(1), 1-16.

Montgomery, Alan, Shibo Li, Kannan Srinivasan, John Liechty. 2004. Modeling Online Browsing and Path Analysis Using Clickstream Data. Marketing Science, 23(4), 579595.

Moon, Sangkil, Wagner Kamakura, Johannes Ledolter. 2007. Estimating Promotion Response When Competitive Promotions Are Unobservable. Journal of Marketing Research, forthcoming.

Mukherji, Prokriti, Shantanu Dutta, Surendra Rajiv. 2004. A Structural Model of Learning for New Products. Working paper, University of Minnesota.

Naik, Prasad, Murali Mantrala, Alan Sawyer. 1998. Planning Media Schedules in the Presence of Dynamic Advertising Quality. Marketing Science, 17(3), 214-235. 
Naik, Prasad, Kalyan Raman, Russell Winer. 2005. Planning Marketing-Mix Strategies in the Presence of Interaction Effects. Marketing Science, 24(1), 25-34.

Narayanan, Sridhar, Puneet Manchanda. 2005. Heterogeneous Learning and the Targeting of Marketing Communications for New Products. Working Paper, University of Chicago.

Narayanan, Sridhar, Puneet Manchanda, Pradeep Chintagunta. 2005. Temporal Differences in the Role of Marketing Communication in New Product Categories. Journal of Marketing Research, 42(3), 278-290.

Netzer, Oded, James Lattin, V. Srinivasan. 2007. A Hidden Markov Model of Customer Relationship Dynamics. Marketing Science, forthcoming.

Newton, Michael, Adrian Raftery. 1994. Approximate Bayesian Inference by the Weighted Likelihood Bootstrap. Journal of the Royal Statistical Society, B, 56(1), 3-48.

Papatla, Purushottam, Lakshman Krishnamurthi. 1996. Measuring the Dynamic Effects of Promotions on Brand Choice. Journal of Marketing Research, 33(1), 20-35.

Pfeifer, Phillip, Robert Carraway. 2000. Modeling Customer Relationships as Markov Chains. Journal of Interactive Marketing, 14(2), 43-55.

Rabiner, Lawrence. 1989. A Tutorial in Hidden Markov Models and Selected Applications in Speech Recognition. Proceedings of the IEEE, 77(2): 257-286.

Roberts, Mary, Paul Berger. 1999. Direct Marketing Management. Prentice-Hall Inc., Upper Saddle River, NJ.

Rossi, Peter, Greg Allenby, Robert McCulloch. 2005. Bayesian Statistics and Marketing. John Wiley \& Sons.

Rossi, Peter, Robert McCulloch, Greg Allenby. 1996. The Value of Purchase History Data in Target Marketing. Marketing Science, 15(4), 321-340.

Rust, Ronald, Tuck Chung. 2006. Marketing Models of Service and Relatioships. Marketing Science, 25(6), 560-580.

Rust, Ronald, Peter Verhoef. 2005. Optimizing the Marketing Interventions Mix in Intermediate-Term CRM. Marketing Science, 24(3), 477-489.

Scott, Steven. 2002. Bayesian Methods for Hidden Markov Models, Recursive Computing in the 21st Century. Journal of the American Statistical Association, 97(475), 337351.

Simester, Duncan, Peng Sun, John Tsitsiklis. 2006. Dynamic Catalog Mailing Policies. Management Science, 52(5), 683-696.

Sismeiro, Catarina, Natalie Mizik, Randolph Bucklin. 2007. Modeling Co-Existing Business Scenarios with Time Series Panel Data. Working paper, Tanaka Business School.

Srinivasan V., R. Kesavan. 1976. An Alternative Interpretation of the Linear Learning Model of Brand Choice. Journal of Consumer Research, 3, 76-83. 